Research article

Open Access

\title{
Synthesis and Antimicrobial Activity of 3-(1,3,4-Oxadiazol-2-yl)quinazolin-4(3H)-ones
}

\author{
Navin B. PateL ${ }^{*}$, Jaymin C. Patel \\ Department of Chemistry, Veer Narmad South Gujarat University, Surat 395007, Gujarat, India. \\ * Corresponding author. E-mail: drnavin@satyam.net.in (N. B. Patel) \\ Sci Pharm. 2010; 78: 171-193 \\ doi:10.3797/scipharm.0912-16 \\ Published: $\quad$ April $26^{\text {th }} 2010$ \\ Accepted: $\quad$ April $26^{\text {th }} 2010$ \\ Received: $\quad$ December $17^{\text {th }} 2009$ \\ This article is available from: http://dx.doi.org/10.3797/scipharm.0912-16 \\ (C) Patel and Patel; licensee Österreichische Apotheker-Verlagsgesellschaft m. b. H., Vienna, Austria. \\ This is an Open Access article distributed under the terms of the Creative Commons Attribution License \\ (http://creativecommons.org/licenses/by/3.0/), which permits unrestricted use, distribution, and reproduction \\ in any medium, provided the original work is properly cited.
}

\begin{abstract}
In attempt to find new pharmacologically active molecules, we report here the synthesis and in vitro antimicrobial activity of various 3-(1,3,4-oxadiazol-2-yl)quinazolin-4(3H)-ones. The antimicrobial activity of title compounds were examined against two gram positive bacteria ( $S$. aureus, $S$. pyogenes), two gram negative bacteria ( $E$. coli, $P$. aeruginosa) and three fungi ( $C$. albicans, $A$. niger, $A$. clavatus) using the broth microdilution method. Some derivatives bearing a bromo or iodo group exhibited very good antimicrobial activity.
\end{abstract}

\section{Keywords}

Antimicrobial activity • 1,3,4-Oxadiazole • Quinazolin-4(3H)-one

\section{Introduction}

The chemistry of heterocyclic compounds has been an interesting field of study for a long time. The synthesis of novel oxadiazole derivatives and investigation of their chemical and biological behavior have gained more importance in recent decades for biological, medicinal and agricultural reasons. 1,3,4-Oxadiazoles represent an important class of heterocyclic compounds. Their derivatives possess a broad spectrum of biological activity in both agrochemicals and pharmaceuticals such as insecticidal, herbicidal, antibacterial, antifungal, analgesic, anti-inflammatory, antimalarial, antiviral, anti-HBV, antianexiety, anticancer, anti-HIV, antitubercular and anticonvulsant [1-14]. Quinazolinone nucleus has been gaining prominence due to the fact that its derivatives have been found to possess wide spectrum of pharmacological properties. Quinazolin-4(3H)-one derivatives are useful heterocycles, possessing potent pharmacological activities such as antibacterial, antifungal, analgesic, anti-inflammatory, anthelminthic, anticancer, anticonvulsant, 
antihistaminic, anti-HIV, antiproliferative, antitubercular, antiviral, CNS depressant, cytotoxicity, diuretic and hypolipidemic [15-30].

1,3,4-Qxadiazoles and quinazolin-4(3H)-ones having various heterocycles possess wide range of pharmacological properties. The aim of the present work was to attach $1,3,4$-oxadiazole residues to quinazolin-4(3H)-one in order to find new biologically active molecules. Thus, the synthesis of novel 1,3,4-oxadiazolyl-quinazolin-4(3H)-one derivatives has been achieved.

\section{Results and Discussion}

\section{Chemistry}

The title compounds $9 \mathbf{a}-\mathbf{f}, \mathbf{1 0 a - f}$ and $12 \mathbf{a}-\mathbf{f}$ were synthesized according to Scheme 2 . The structure of all the synthesized compounds were evaluated by spectral data. Benzoic acid derivatives $\mathbf{1} \mathbf{a}, \mathbf{b}$ were converted in to esters $\mathbf{2} \mathbf{a}, \mathbf{b}$ using methanol and catalytic amount of sulphuric acid. Esters $\mathbf{2 a , b}$ on treatment with hydrazine hydrate yielded corresponding hydrazides $\mathbf{3} \mathbf{a}, \mathbf{b}$. The IR spectra of $\mathbf{3} \mathbf{a}, \mathbf{b}$ showed the absence of ester stretching frequency, instead in gave a band at around $1650 \mathrm{~cm}^{-1}$ for carbonyl group and showing sharp bands in the region of $3300-3435 \mathrm{~cm}^{-1}$ due to $-\mathrm{NHNH}_{2}$ group. Hydrazides on cyclization reaction with methanolic cyanogen bromide and 4-aminobenzoic acid in phosphorus oxychloride yielded amino substituted 1,3,4-oxadiazoles $\mathbf{4 a , b}$ and $\mathbf{5 a , b}$ respectively (Scheme 1). 1,3,4-oxadiazole showed $\mathrm{C}=\mathrm{N}$ stretching at around $1655 \mathrm{~cm}^{-1}$ and $\mathrm{C}-\mathrm{O}-\mathrm{C}$ stretching at around $1270 \mathrm{~cm}^{-1}$ and $1040 \mathrm{~cm}^{-1}$. Signals at around $158 \delta \mathrm{ppm}$ and $164 \delta$ ppm confirmed the C-2 and C-5 carbon of 1,3,4-oxadiazole unite.<smiles>[R]c1ccccc1C(=O)O</smiles>

$1 a, b$

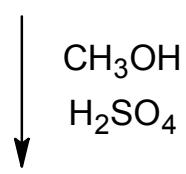<smiles>[R]c1ccccc1C(=O)OC</smiles>

2a,b<smiles>[R]c1cccc(-c2nnc(-c3ccc(N)cc3)o2)c1</smiles>

$5 a, b$<smiles>Nc1ccc(C(=O)O)cc1</smiles><smiles>[R]c1ccccc1C(N)=O</smiles>

3a,b

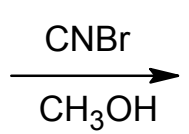<smiles>CCCC</smiles>

$\mathrm{R}^{1}=2-\mathrm{Cl}, 4-\mathrm{Cl}$

Sch. 1. 

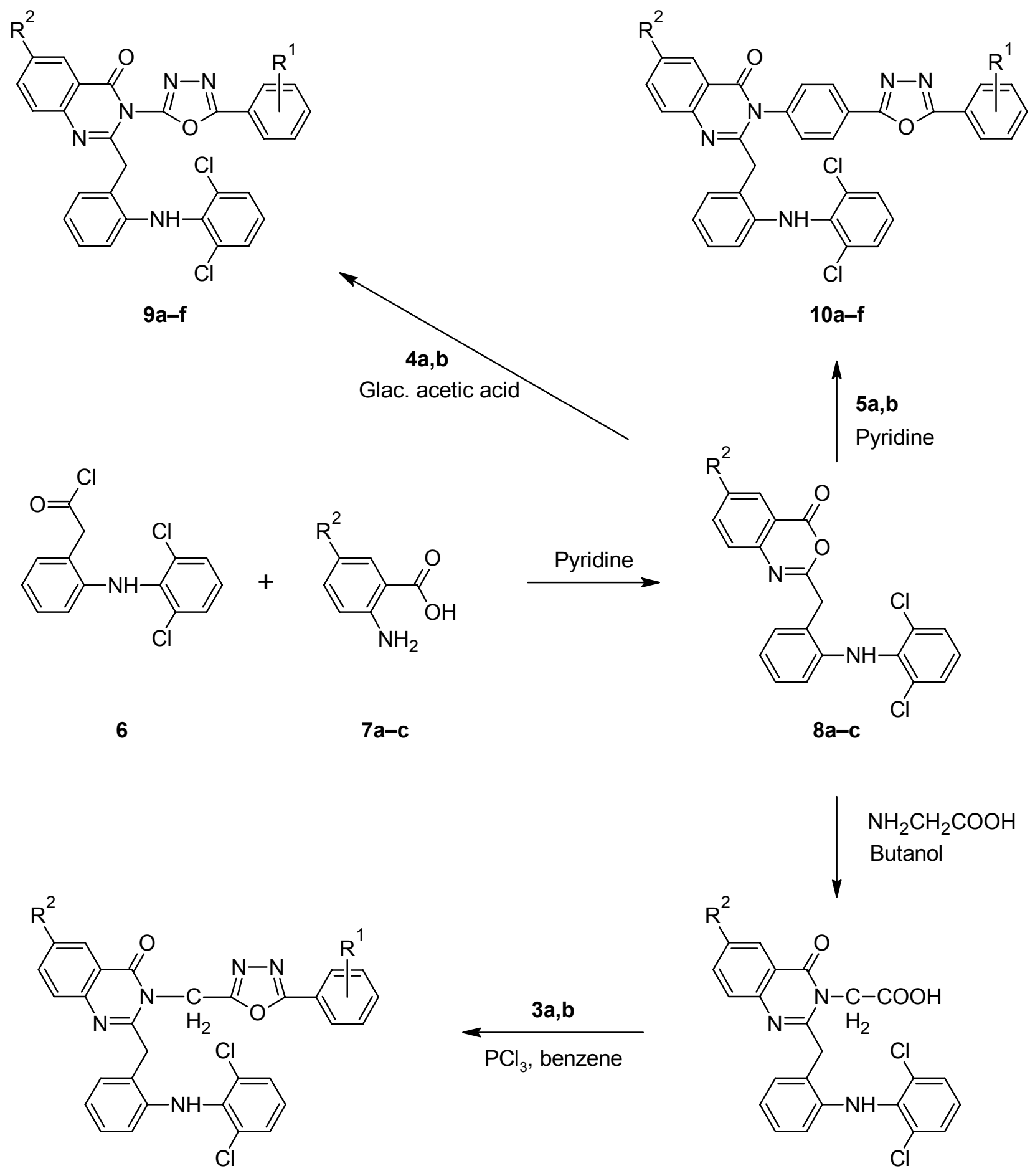

$12 a-f$
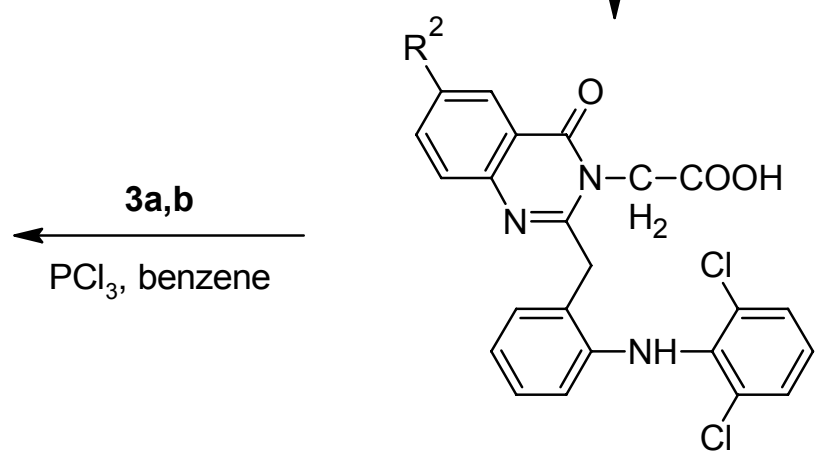

$11 \mathrm{a}-\mathrm{c}$

$\mathrm{R}^{1}=2-\mathrm{Cl}, 4-\mathrm{C}$

$\mathrm{R}^{2}=\mathrm{H}, \mathrm{Br}, \mathrm{I}$

Sch. 2. 
Substituted benzoxazinones 8a-c were prepared by reaction of acid chloride $\mathbf{6}$ with substituted anthranilic acid $\mathbf{7 a - c}$ in pyridine. Then condensation reaction of $\mathbf{8 a}-\mathbf{c}$ with 1,3,4-oxadiazoles $\mathbf{4 a , b}$ and $\mathbf{5 a} \mathbf{a} \mathbf{b}$ yielded the desired compounds $\mathbf{9} \mathbf{a}-\mathbf{f}$ and $\mathbf{1 0} \mathbf{a}-\mathbf{f}$. Substituted benzoxazinones $\mathbf{8 a - c}$ on reaction with glycine yielded quinazolin-4(3H)-ones $11 \mathbf{a}-\mathbf{c}$ which on cyclization reaction with hydrazides $\mathbf{3 a , b}$ gave the desired compounds 12a-f(Scheme 2). IR spectra showed $C=O$ and $C=N$ stretching frequencies of quinazolinone at around $1680 \mathrm{~cm}^{-1}$ and $1610 \mathrm{~cm}^{-1}$ respectively, further confirmed by ${ }^{13} \mathrm{C}$ NMR spectra, which showed $\mathrm{C}=\mathrm{O}$ and $\mathrm{C}=\mathrm{N}$ signal at around $160.5 \delta \mathrm{ppm}$ and $163.5 \delta$ ppm respectively.

\section{Antibacterial activity}

Tab. 1. Antibacterial activity (MBC, $\mu \mathrm{g} / \mathrm{ml})$ of compounds $4 \mathbf{a}, \mathbf{b}, \mathbf{5 a}, \mathbf{b}, \mathbf{9 a}-\mathbf{f}, \mathbf{1 0} \mathbf{a}-\mathbf{f}$ and $12 a-f$.

\begin{tabular}{|c|c|c|c|c|c|c|}
\hline \multirow[b]{2}{*}{ Comp. } & \multirow[b]{2}{*}{$\mathbf{R}^{1}$} & \multirow[b]{2}{*}{$\mathbf{R}^{2}$} & \multicolumn{2}{|c|}{ Gram positive bacteria } & \multicolumn{2}{|c|}{ Gram negative bacteria } \\
\hline & & & $\begin{array}{l}\text { S. aureus } \\
\text { MTCC } 96\end{array}$ & $\begin{array}{l}\text { S. pyogenes } \\
\text { MTCC } 442\end{array}$ & $\begin{array}{c}\text { E. coli } \\
\text { MTCC } 443\end{array}$ & $\begin{array}{c}\text { P. aeruginosa } \\
\text { MTCC } 1688\end{array}$ \\
\hline $4 a$ & $2-\mathrm{Cl}$ & - & 250 & 250 & 50 & 250 \\
\hline $4 b$ & $4-\mathrm{Cl}$ & - & 200 & 250 & 250 & 500 \\
\hline $5 \mathbf{a}$ & $2-\mathrm{Cl}$ & - & 100 & 150 & 250 & 200 \\
\hline $5 b$ & $4-\mathrm{Cl}$ & - & 200 & 250 & 500 & 500 \\
\hline $9 a$ & $2-\mathrm{Cl}$ & $\mathrm{H}$ & 250 & 200 & 500 & 500 \\
\hline $9 b$ & $2-\mathrm{Cl}$ & $\mathrm{Br}$ & 100 & 250 & 200 & 250 \\
\hline 9c & $2-\mathrm{Cl}$ & I & 500 & 500 & 100 & 250 \\
\hline 9d & $4-\mathrm{Cl}$ & $\mathrm{H}$ & 200 & 250 & 200 & 250 \\
\hline $9 e$ & $4-\mathrm{Cl}$ & $\mathrm{Br}$ & 250 & 250 & 250 & 150 \\
\hline $9 f$ & $4-\mathrm{Cl}$ & I & 500 & 1000 & 250 & 200 \\
\hline $10 a$ & $2-\mathrm{Cl}$ & $\mathrm{H}$ & 150 & 250 & 500 & 500 \\
\hline $10 b$ & $2-\mathrm{Cl}$ & $\mathrm{Br}$ & 500 & 1000 & 250 & 500 \\
\hline $10 c$ & $2-\mathrm{Cl}$ & 1 & 100 & 150 & 150 & 500 \\
\hline $10 d$ & $4-\mathrm{Cl}$ & $\mathrm{H}$ & 500 & 500 & 200 & 250 \\
\hline $10 e$ & $4-\mathrm{Cl}$ & $\mathrm{Br}$ & 250 & 250 & 150 & 250 \\
\hline $10 f$ & $4-\mathrm{Cl}$ & I & 62.5 & 150 & 100 & 250 \\
\hline $12 a$ & $2-\mathrm{Cl}$ & $\mathrm{H}$ & 500 & 500 & 250 & 250 \\
\hline $12 b$ & $2-\mathrm{Cl}$ & $\mathrm{Br}$ & 500 & 500 & 100 & 50 \\
\hline $12 c$ & $2-\mathrm{Cl}$ & I & 50 & 250 & 125 & 200 \\
\hline $12 d$ & $4-\mathrm{Cl}$ & $\mathrm{H}$ & 500 & 500 & 150 & 200 \\
\hline $12 e$ & $4-\mathrm{Cl}$ & $\mathrm{Br}$ & 500 & 250 & 100 & 250 \\
\hline $12 f$ & $4-\mathrm{Cl}$ & I & 200 & 200 & 50 & 150 \\
\hline Ampicillin & - & - & 250 & 100 & 100 & 100 \\
\hline
\end{tabular}

The minimal bactericidal concentrations of the tested compounds are shown in Tab. 1. The three different series of 1,3,4-oxadiazolyl-quinazolin-4(3H)-ones $\mathbf{9 a - f}, \mathbf{1 0} \mathbf{a}-\mathbf{f}$ and $\mathbf{1 2 a - f}$ were tested for in vitro antibacterial activity against two gram positive bacteria ( $S$. aureus MTCC 96, S. pyogenes MTCC 442) and two gram negative bacteria (E. coli MTCC 443, $P$. aeruginosa MTCC 1688). Ampicillin was used as a standard drug. Results showed that, 
most of the compounds possessed very good antibacterial activity (MBC $=50-250 \mu \mathrm{g} / \mathrm{ml})$ against gram positive bacteria $S$. aureus. Some of the compounds possessed excellent activity as compared to ampicillin. Compounds 5a, 9b, 10a, 10c, 10f and 12c showed MBC value in the range between $50-150 \mu \mathrm{g} / \mathrm{ml}$ while standard drug ampicillin itself had MBC value of $250 \mu \mathrm{g} / \mathrm{ml}$ against gram positive bacteria $S$. aureus. Compounds $\mathbf{4 a}, \mathbf{4 b}, \mathbf{5 b}$, 9a, 9d, 9e, 10e and $12 \mathrm{f}$ imparted parallel activity as ampicillin with MBC in the range 200$250 \mu \mathrm{g} / \mathrm{ml}$. 5a, 10c and $10 \mathrm{f}$ have $\mathrm{MBC}$ at $150 \mu \mathrm{g} / \mathrm{ml}$ which were comparatively good as ampicillin against $S$. pyogenes. $4 a$ and $12 \mathrm{f}$ showed excellent activity at $50 \mu \mathrm{g} / \mathrm{ml}$ while 9c, $10 f, 12 b$ and $12 \mathrm{e}$ possessed good activity at $100 \mu \mathrm{g} / \mathrm{ml}$ against gram negative bacteria $E$. coli as compared to ampicillin. Compound $12 \mathrm{~b}$ showed excellent activity at $50 \mu \mathrm{g} / \mathrm{ml}$, while 9e and $12 \mathrm{f}$ exhibited good activity at $150 \mu \mathrm{g} / \mathrm{ml}$ against $P$. aeruginosa. The remaining compounds of the three different series possessed moderate to poor activities.

\section{Antifungal activity}

Tab. 2. Antifungal activity (MFC, $\mu \mathrm{g} / \mathrm{ml}$ ) of compds. $4 \mathbf{a}, \mathbf{b}, \mathbf{5 a}, \mathbf{b}, \mathbf{9 a}-\mathbf{f}, \mathbf{1 0 a - f}$ and $12 \mathbf{a}-\mathbf{f}$.

\begin{tabular}{|c|c|c|c|c|c|}
\hline \multirow[b]{2}{*}{ Comp. } & \multirow[b]{2}{*}{$\mathbf{R}^{1}$} & \multirow[b]{2}{*}{$\mathbf{R}^{2}$} & \multicolumn{3}{|c|}{$\begin{array}{c}\text { Fungal species } \\
\end{array}$} \\
\hline & & & $\begin{array}{l}\text { C. albicans } \\
\text { MTCC } 227\end{array}$ & $\begin{array}{c}\text { A. niger } \\
\text { MTCC } 282\end{array}$ & $\begin{array}{l}\text { A. clavatus } \\
\text { MTCC } 1323\end{array}$ \\
\hline $4 a$ & $2-\mathrm{Cl}$ & - & 250 & 1000 & 1000 \\
\hline 4b & $4-\mathrm{Cl}$ & - & $>1000$ & $>1000$ & $>1000$ \\
\hline $5 \mathbf{a}$ & $2-\mathrm{Cl}$ & - & 500 & 500 & 500 \\
\hline $5 b$ & $4-\mathrm{Cl}$ & - & 500 & $>1000$ & $>1000$ \\
\hline 9a & $2-\mathrm{Cl}$ & $\mathrm{H}$ & 500 & 250 & 250 \\
\hline $9 b$ & $2-\mathrm{Cl}$ & $\mathrm{Br}$ & 500 & 500 & 500 \\
\hline 9c & $2-\mathrm{Cl}$ & 1 & 500 & 500 & $>1000$ \\
\hline 9d & $4-\mathrm{Cl}$ & $\mathrm{H}$ & 500 & 250 & 250 \\
\hline $9 e$ & $4-\mathrm{Cl}$ & $\mathrm{Br}$ & 500 & 500 & 500 \\
\hline $9 f$ & $4-\mathrm{Cl}$ & I & 250 & 200 & 200 \\
\hline $10 a$ & $2-\mathrm{Cl}$ & $\mathrm{H}$ & $>1000$ & $>1000$ & $>1000$ \\
\hline $10 b$ & $2-\mathrm{Cl}$ & $\mathrm{Br}$ & 250 & 500 & $>1000$ \\
\hline $10 c$ & $2-\mathrm{Cl}$ & 1 & $>1000$ & $>1000$ & $>1000$ \\
\hline $10 d$ & $4-\mathrm{Cl}$ & $\mathrm{H}$ & 500 & $>1000$ & $>1000$ \\
\hline $10 e$ & $4-\mathrm{Cl}$ & $\mathrm{Br}$ & 500 & $>1000$ & $>1000$ \\
\hline $10 f$ & $4-\mathrm{Cl}$ & I & 250 & 500 & 500 \\
\hline $12 a$ & $2-\mathrm{Cl}$ & $\mathrm{H}$ & $>1000$ & 500 & $>1000$ \\
\hline $12 b$ & $2-\mathrm{Cl}$ & $\mathrm{Br}$ & 250 & 500 & 250 \\
\hline $12 c$ & $2-\mathrm{Cl}$ & I & 100 & 200 & 200 \\
\hline $12 d$ & $4-\mathrm{Cl}$ & $\mathrm{H}$ & $>1000$ & $>1000$ & $>1000$ \\
\hline $12 e$ & $4-\mathrm{Cl}$ & $\mathrm{Br}$ & 500 & $>1000$ & 500 \\
\hline $12 f$ & $4-\mathrm{Cl}$ & 1 & 200 & $>1000$ & 500 \\
\hline Griseofulvin & - & - & 500 & 100 & 100 \\
\hline
\end{tabular}

Minimal fungicidal concentrations of the synthesized compounds are shown in Table 2. For in vitro antifungal activity, three fungal species C. albicans MTCC 227, A. niger MTCC 282 and $A$. clavatus MTCC 1323 were used and compared with standard drug griseofulvin. Most of the compounds possessed very good antifungal activity against $C$. albicans. Their 
MFC values are in the range between $100-500 \mu \mathrm{g} / \mathrm{ml}$. Compound 12c showed excellent activity at $100 \mu \mathrm{g} / \mathrm{ml}$ whereas compounds $\mathbf{4 a}, \mathbf{9 f}, \mathbf{1 0 b}, \mathbf{1 0 f}, \mathbf{1 2 b}$ and $\mathbf{1 2 f}$ possessed very good activity at 200-250 $\mathrm{\mu g} / \mathrm{ml}$ while $\mathbf{5 a , b}, \mathbf{9 a - e , 1 0 d , e ~ a n d ~} \mathbf{1 2 e}$ share similar activities as griseofulvin which was $500 \mu \mathrm{g} / \mathrm{ml}$ against $C$. albicans. Compounds 9a, 9d, 9f and 12c showed moderate activities with MFC of $200-250 \mu \mathrm{g} / \mathrm{ml}$ against $A$. niger and $A$. clavatus. The remaining compounds displayed poor activities against both fungal species $A$. niger and $A$. clavatus as compared to control drug griseofulvin.

\section{Experimental}

\section{Chemistry}

All chemical were of analytical grade and used directly. Melting points were determined in PMP-DM scientific melting point apparatus and are uncorrected. IR spectra were recorded on a Perkin-Elmer RX 1 FTIR spectrophotometer, using potassium bromide pellets and the frequencies are expressed in $\mathrm{cm}^{-1}$. The ${ }^{1} \mathrm{H}$ NMR and ${ }^{13} \mathrm{C}$ NMR spectra were recorded with a Bruker Avance II 400 NMR spectrometer, using tetramethylsilane as the internal reference, with dimethylsulphoxide $D M S O-d_{6}$ as solvent. The chemical shifts are reported in parts per million (ppm). Elemental analyses were performed on a Heraeus Carlo Erba $1180 \mathrm{CHN}$ analyzer. The purity of compounds was confirmed by TLC using Merck silica gel 60 F254 plates using toluene:ethylacetate:methanol $(7: 2: 1)$ as a mobile phase and spots were visualized under UV radiation. $\{2-[(2,6-$ Dichlorophenyl)amino]phenyl\}acetyl chloride (6) was synthesized by the literature procedure [31].

\section{General procedure for esters $(2 a, b)$}

Substituted benzoic acid $\mathbf{1} \mathbf{a}, \mathbf{b}(20 \mathrm{mmol})$ and $30 \mathrm{ml}$ methanol was refluxed on water bath for 5-8 $\mathrm{h}$ in a few drops of concentrated sulfuric acid as a catalyst. After completion of the reaction, it was poured onto ice cold water. The obtained solid was washed with sodium bicarbonate solution (5\%), dried and recrystallized twice from methanol.

Methyl 2-chlorobenzoate (2a)

Yield: $75 \%$, bp: $232-236^{\circ} \mathrm{C}$. IR (KBr), v, cm${ }^{-1}: 740(\mathrm{C}-\mathrm{Cl}), 2856,2960\left(\mathrm{CH}_{3}\right), 1286,1121$ $(\mathrm{C}-\mathrm{O}-\mathrm{C}), 1722(\mathrm{C}=\mathrm{O})$.

Methyl 4-chlorobenzoate (2b)

Yield: $72 \%, \mathrm{mp}: 40-45^{\circ} \mathrm{C}$. IR (KBr), v, $\mathrm{cm}^{-1}: 742(\mathrm{C}-\mathrm{Cl}), 2852,2959\left(\mathrm{CH}_{3}\right), 1282,1119(\mathrm{C}-$ $\mathrm{O}-\mathrm{C}), 1720(\mathrm{C}=\mathrm{O})$.

\section{General procedure for hydrazides $(3 a, b)$}

To a solution of methyl benzoates $2 \mathbf{a}, \mathbf{b}(10 \mathrm{mmol})$ in $15 \mathrm{ml}$ methanol was added hydrazine hydrate $(20 \mathrm{mmol})$. The reaction mixture was refluxed on a water bath for $8-10 \mathrm{~h}$ and allowed to stand overnight. The crystals formed were filtered, washed and after drying recrystallized from methanol.

\section{2-Chlorobenzohydrazide (3a)}

Yield: $70 \%, \mathrm{mp}: 112-116^{\circ} \mathrm{C}$. IR (KBr), v, cm ${ }^{-1}: 742$ (C-Cl), 1650 (C=O), $3308(\mathrm{NH}), 3429$, $3348\left(\mathrm{NH}_{2}\right)$. 


\section{4-Chlorobenzohydrazide (3b)}

Yield: 67\%, mp: $163-166^{\circ} \mathrm{C}$. IR (KBr), v, cm ${ }^{-1}: 745$ (C-Cl), 1645 (C=O), $3310(\mathrm{NH}), 3433$, $3352\left(\mathrm{NH}_{2}\right)$.

\section{General procedure for 5-substituted phenyl-1,3,4-oxadiazol-2-amines (4a,b)}

To the $10 \mathrm{ml}$ methanolic solution of substituted benzohydrazides $\mathbf{3 a , b}(5 \mathrm{mmol})$, cyanogen bromide $(7.5 \mathrm{mmol})$ was added. The reaction mixture was refluxed on water bath for 5-7 $\mathrm{h}$. The resulting solution was cooled and neutralized with sodium bicarbonate solution $(5 \% \mathrm{w} / \mathrm{v})$. The solid thus separated out was filtered, washed with water, dried and recrystallized from methanol.

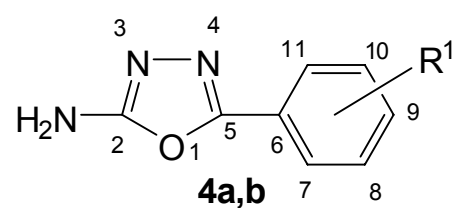

Fig. 1. Numbering of 5-substituted phenyl-1,3,4-oxadiazol-2-amines $\mathbf{4 a , b}$

5-(2-Chlorophenyl)-1,3, 4-oxadiazol-2-amine (4a)

Yield: $62 \%$, mp: $165-168^{\circ} \mathrm{C}$, lit. $164-166^{\circ} \mathrm{C}$ [32]. IR (KBr), v, $\mathrm{cm}^{-1}: 742$ (C-Cl), 1265, 1036 (C-O-C), $1649(\mathrm{C}=\mathrm{N}), 3470,3402\left(\mathrm{NH}_{2}\right) .{ }^{1} \mathrm{H}$ NMR (400 MHz, DMSO-d, TMS): $\delta 7.35$ (bs, $\left.2 \mathrm{H}, \mathrm{NH}_{2}\right), 7.49(\mathrm{t}, J=7.52 \mathrm{~Hz}, 1 \mathrm{H}, \mathrm{H}-9), 7.55(\mathrm{t}, J=7.36 \mathrm{~Hz}, 1 \mathrm{H}, \mathrm{H}-10), 7.65$ (d, $J=7.8$ $\mathrm{Hz}, 1 \mathrm{H}, \mathrm{H}-8), 7.70$ (d, $J=7.48 \mathrm{~Hz}, 1 \mathrm{H}, \mathrm{H}-11) .{ }^{13} \mathrm{C}$ NMR (100 MHz, DMSO-d 6 , TMS): $\delta$ 127.53 (C-10), 128.12 (C-11), 128.69 (C-8), 129.81 (C-9), 131.43 (C-7), 135.18 (C-6), 157.53 (C-2), 163.50 (C-5). Anal. Calcd. for $\mathrm{C}_{8} \mathrm{H}_{6} \mathrm{~N}_{3} \mathrm{OCl}: \mathrm{C}, 49.12 ; \mathrm{H}, 3.09 ; \mathrm{N}, 21.48$. Found: C, 49.25; H, 3.14; N, 21.41.

\section{5-(4-Chlorophenyl)-1,3,4-oxadiazol-2-amine (4b)}

Yield: $70 \%$, mp: $243-245^{\circ} \mathrm{C}$, lit. $231-233^{\circ} \mathrm{C}$ [32]. IR (KBr), v, $\mathrm{cm}^{-1}: 745$ (C-Cl), 1277, 1043 (C-O-C), $1662(\mathrm{C}=\mathrm{N}), 3480,3396\left(\mathrm{NH}_{2}\right) .{ }^{1} \mathrm{H}$ NMR (400 MHz, DMSO-d, TMS): $\delta 7.37$ (bs, $\left.2 \mathrm{H}, \mathrm{NH}_{2}\right), 7.62(\mathrm{~d}, J=8.24 \mathrm{~Hz}, 2 \mathrm{H}, \mathrm{H}-8,10), 7.69(\mathrm{~d}, J=8.24 \mathrm{~Hz}, 2 \mathrm{H}, \mathrm{H}-7,11) .{ }^{13} \mathrm{C}$ NMR (100 MHz, DMSO-d $d_{6}$, TMS): $\delta 123.34$ (C-6), 131.78 (C-7,11), 133.20 (8,10), 135.42 (C-9), 157.58 (C-2), 163.56 (C-5). Anal. Calcd. for $\mathrm{C}_{8} \mathrm{H}_{6} \mathrm{~N}_{3} \mathrm{OCl}$ : C, 49.12; $\mathrm{H}, 3.09 ; \mathrm{N}, 21.48$. Found: C, 48.98; H, 3.17; N, 21.56.

\section{General procedure for 4-(5-substitutedphenyl-1,3,4-oxadiazol-2-yl)benzenamines $(5 a, b)$}

A mixture of 4-aminobenzoic acid $(5 \mathrm{mmol})$ and substituted benzohydrazides $3 \mathbf{a}, \mathbf{b}$ $(5 \mathrm{mmol})$ in $5 \mathrm{ml}$ phosphorus oxychloride was refluxed on water bath for $7-10 \mathrm{~h}$. After the completion of reaction, it was cooled and poured onto crushed ice with continuous stirring. The solid mass separated was neutralized with sodium bicarbonate solution $(10 \% \mathrm{w} / \mathrm{v})$. The resulting solid thus obtained was collected by filtration, washed well with cold water, dried and recrystallized from absolute ethanol. 


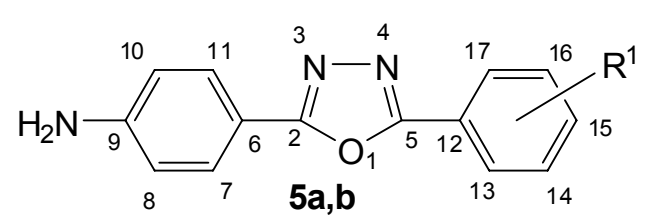

Fig. 2. Numbering of 4-(5-substituted phenyl-1,3,4-oxadiazol-2-yl)benzenamines 5a,b

\section{4-[5-(2-Chlorophenyl)-1,3,4-oxadiazol-2-yl]benzenamine (5a)}

Yield: $63 \%$, mp: $175-178^{\circ} \mathrm{C}$. IR (KBr), v, cm${ }^{-1}: 746$ (C-Cl), 1266, 1042 (C-O-C), 1653 $(\mathrm{C}=\mathrm{N}), 3482,3380\left(\mathrm{NH}_{2}\right) .{ }^{1} \mathrm{H}$ NMR $\left(400 \mathrm{MHz}, \mathrm{DMSO}-d_{6}, \mathrm{TMS}\right): \delta 5.46\left(\mathrm{bs}, 2 \mathrm{H}, \mathrm{NH}_{2}\right), 6.80$ (d, J = 8.32 Hz, 2H, H-8,10), 7.31 (d, J = 8.32 Hz, 2H, H-7,11), 7.50 (t, J = $7.56 \mathrm{~Hz}, 1 \mathrm{H}, \mathrm{H}-$ 15), 7.56 (t, $J=7.4 \mathrm{~Hz}, 1 \mathrm{H}, \mathrm{H}-16), 7.64(\mathrm{~d}, J=7.84 \mathrm{~Hz}, 1 \mathrm{H}, \mathrm{H}-14), 7.68(\mathrm{~d}, J=7.5 \mathrm{~Hz}, 1 \mathrm{H}$, $\mathrm{H}-17) \cdot{ }^{13} \mathrm{C}$ NMR $\left(100 \mathrm{MHz}\right.$, DMSO-d $\left.d_{6}, \mathrm{TMS}\right): \delta 108.13$ (C-6), 114.84 (C-8,10), 127.53 (C16), 128.14 (C-17), 128.62 (C-7,11), 128.74 (C-14), 129.85 (C-15), 131.38 (C-13), 135.22 (C-12), 148.48 (C-9), $162.37(\mathrm{C}-2,5)$. Anal. Calcd. for $\mathrm{C}_{14} \mathrm{H}_{10} \mathrm{~N}_{3} \mathrm{OCl}$ : C, 61.89; $\mathrm{H}, 3.71 ; \mathrm{N}$, 15.47. Found: C, $61.76 ; \mathrm{H}, 3.75 ; \mathrm{N}, 15.39$.

\section{4-[5-(4-Chlorophenyl)-1,3,4-oxadiazol-2-yl]benzenamine (5b)}

Yield: $65 \%$, mp: $210-214^{\circ} \mathrm{C}$, lit. $210-212^{\circ} \mathrm{C}$ [12]. IR (KBr), v, cm ${ }^{-1}: 743$ (C-Cl), 1268, 1040 (C-O-C), $1658(\mathrm{C}=\mathrm{N}), 3482,3375\left(\mathrm{NH}_{2}\right) .{ }^{1} \mathrm{H}$ NMR (400 MHz, DMSO-d 6 , TMS): $\delta 5.45$ (bs, $\left.2 \mathrm{H}, \mathrm{NH}_{2}\right), 6.79(\mathrm{~d}, J=8.36 \mathrm{~Hz}, 2 \mathrm{H}, \mathrm{H}-8,10), 7.29(\mathrm{~d}, J=8.36 \mathrm{~Hz}, 2 \mathrm{H}, \mathrm{H}-7,11), 7.63(\mathrm{~d}, J=$ $8.2 \mathrm{~Hz}, 2 \mathrm{H}, \mathrm{H}-14,16), 7.68$ (d, $J=8.2 \mathrm{~Hz}, 2 \mathrm{H}, \mathrm{H}-13,17) .{ }^{13} \mathrm{C}$ NMR $(100 \mathrm{MHz}$, DMSO-d TMS): $\delta 107.42$ (C-6), 114.45 (C-8,10), 123.31 (C-12), 128.73 (C-7,11), 131.82 (C-13,17), 133.24 (C-14,16), 135.44 (C-15), 148.53 (C-9), 161.89 (C-2,5). Anal. Calcd. for $\mathrm{C}_{14} \mathrm{H}_{10} \mathrm{~N}_{3} \mathrm{OCl}: \mathrm{C}, 61.89 ; \mathrm{H}, 3.71 ; \mathrm{N}, 15.47$. Found: $\mathrm{C}, 61.78 ; \mathrm{H}, 3.66 ; \mathrm{N}, 15.54$.

\section{General procedure for benzoxazinones (8a-c)}

A mixture of $\{2-[(2,6$-Dichlorophenyl)amino]phenyl\}acetyl chloride (6) $(10 \mathrm{mmol})$ and substituted anthranilic acids $7 \mathrm{a}-\mathrm{c}(10 \mathrm{mmol})$ in $20 \mathrm{ml}$ pyridine were stirred at $0-5^{\circ} \mathrm{C}$ for $1 \mathrm{~h}$, further stirred for $1 \mathrm{~h}$ at room temperature. After completion of reaction, a pasty mass obtained, was washed thoroughly with sodium bicarbonate $(5 \% \mathrm{w} / \mathrm{v})$ to remove unreacted acid. A solid separated was filtered, dried and recrystallized from methanol.

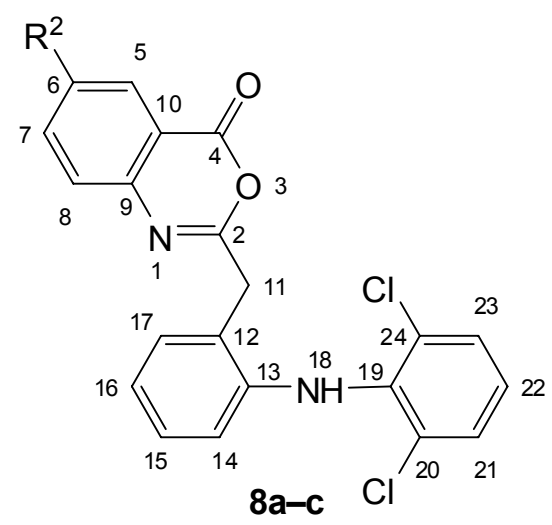

Fig. 3. Numbering of Benzoxazinones 8a-c 


\section{2-[2-(2,6-Dichlorophenyl)amino]benzyl-4H-3,1-benzoxazin-4-one (8aF}

Yield: $53 \%$, mp: $183-186^{\circ} \mathrm{C}$, lit. $185-187^{\circ} \mathrm{C}$ [33]. IR (KBr), v, cm ${ }^{-1}: 745$ (C-Cl), 1151 (CO), $1316(\mathrm{C}-\mathrm{N}), 1620(\mathrm{C}=\mathrm{N}), 1742(\mathrm{C}=\mathrm{O}), 2925,2851\left(\mathrm{CH}_{2}\right), 3449(\mathrm{NH}) .{ }^{1} \mathrm{H}$ NMR $(400$ $\left.\mathrm{MHz}, \mathrm{DMSO}-d_{6}, \mathrm{TMS}\right): \delta 3.52(\mathrm{~s}, 2 \mathrm{H}, \mathrm{H}-11), 6.39(\mathrm{~d}, J=7.96 \mathrm{~Hz}, 1 \mathrm{H}, \mathrm{H}-14), 6.88(\mathrm{t}, \mathrm{J}=$ $7.4 \mathrm{~Hz}, 1 \mathrm{H}, \mathrm{H}-16), 7.04-7.09(\mathrm{~m}, 2 \mathrm{H}, \mathrm{H}-15,22), 7.21(\mathrm{~d}, J=7.54 \mathrm{~Hz}, 1 \mathrm{H}, \mathrm{H}-17), 7.42(\mathrm{~d}, J=$ $8.08 \mathrm{~Hz}, 2 \mathrm{H}, \mathrm{H}-21,23), 7.51(\mathrm{~d}, J=8.12 \mathrm{~Hz}, 1 \mathrm{H}, \mathrm{H}-8), 7.84(\mathrm{t}, J=7.8 \mathrm{~Hz}, 1 \mathrm{H}, \mathrm{H}-7), 8.06(\mathrm{t}$, $J=7.64 \mathrm{~Hz}, 1 \mathrm{H}, \mathrm{H}-6), 8.12(\mathrm{~d}, J=7.72 \mathrm{~Hz}, 1 \mathrm{H}, \mathrm{H}-5), 9.12$ (bs, $1 \mathrm{H}, \mathrm{H}-18) .{ }^{13} \mathrm{C}$ NMR $(100$ MHz, DMSO- $\left.d_{6}, \mathrm{TMS}\right): \delta 32.47$ (C-11), 116.27 (C-16), 116.54 (C-10), 120.54 (C-14), 122.35 (C-8), 124.15 (C-22), 126.61 (C-15), 127.12 (C-12), 127.32 (C-21,23), 127.54 (C6), 129.34 (C-20,24), 131.23 (C-17), 131.52 (C-5), 135.43 (C-7), 137.23 (C-19), 141.76 (C13), 149.53 (C-9), 159.36 (C-4), 164.51 (C-2). Anal. Calcd. for $\mathrm{C}_{21} \mathrm{H}_{14} \mathrm{Cl}_{2} \mathrm{~N}_{2} \mathrm{O}_{2}$ : C, 63.49; $\mathrm{H}$, $3.55 ;$ N, 7.05. Found: C, 63.45; H, 3.56; N, 7.03.

\section{6-Bromo-2-[2-(2,6-dichlorophenyl)amino]benzyl-4H-3,1-benzoxazin-4-one (8b)}

Yield: 55\%, mp: $194-198^{\circ} \mathrm{C}$, lit. 193-196 ${ }^{\circ} \mathrm{C}$. [34]. IR (KBr), v, cm ${ }^{-1}: 565$ (C-Br), 743 (C$\mathrm{Cl}), 1153(\mathrm{C}-\mathrm{O}), 1318(\mathrm{C}-\mathrm{N}), 1618(\mathrm{C}=\mathrm{N}), 1740(\mathrm{C}=\mathrm{O}), 2926,2850\left(\mathrm{CH}_{2}\right), 3446(\mathrm{NH}) .{ }^{1} \mathrm{H}$

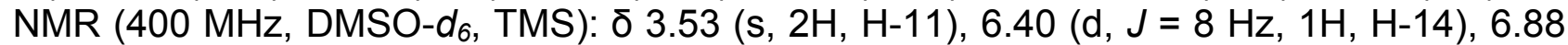
$(\mathrm{t}, J=7.44 \mathrm{~Hz}, 1 \mathrm{H}, \mathrm{H}-16), 7.03-7.08(\mathrm{~m}, 2 \mathrm{H}, \mathrm{H}-15,22), 7.22(\mathrm{~d}, J=7.58 \mathrm{~Hz}, 1 \mathrm{H}, \mathrm{H}-17)$, $7.41(\mathrm{~d}, J=8.16 \mathrm{~Hz}, 2 \mathrm{H}, \mathrm{H}-21,23), 7.65$ (d, $J=8.32 \mathrm{~Hz}, 1 \mathrm{H}, \mathrm{H}-8), 8.12(\mathrm{~d}, J=8.32 \mathrm{~Hz}, 1 \mathrm{H}$, $\mathrm{H}-7), 8.16$ (s, 1H, H-5), 9.10 (bs, $1 \mathrm{H}, \mathrm{H}-18) .{ }^{13} \mathrm{C}$ NMR (100 MHz, DMSO-d, TMS): $\delta 32.43$ (C-11), 116.31 (C-16), 118.64 (C-10), 120.62 (C-14), 121.67 (C-6), 124.31 (C-22), 124.57 (C-8), 126.54 (C-15), 127.17 (C-12), 127.43 (C-21,23), 129.41 (C-20,24), 131.12 (C-17), 135.22 (C-5), 137.29 (C-19), 138.23 (C-7), 141.78 (C-13), 148.73 (C-9), 159.23 (C-4), 164.33 (C-2). Anal. Calcd. for $\mathrm{C}_{21} \mathrm{H}_{13} \mathrm{BrCl}_{2} \mathrm{~N}_{2} \mathrm{O}_{2}$ : C, 52.97; $\mathrm{H}, 2.75 ; \mathrm{N}, 5.88$. Found: C, $52.94 ; \mathrm{H}, 2.74 ; \mathrm{N}, 5.90$.

\section{2-[2-(2,6-Dichlorophenyl)amino]benzyl-6-iodo-4H-3,1-benzoxazin-4-one (8c)}

Yield: $58 \%$, mp: $189-193^{\circ} \mathrm{C}$. IR (KBr), v, cm ${ }^{-1}: 620$ (C-I), 747 (C-Cl), 1148 (C-O), 1320 (C$\mathrm{N}), 1617(\mathrm{C}=\mathrm{N}), 1745(\mathrm{C}=\mathrm{O}), 2923,2848\left(\mathrm{CH}_{2}\right), 3450(\mathrm{NH}) .{ }^{1} \mathrm{H}$ NMR $\left(400 \mathrm{MHz}, \mathrm{DMSO}-d_{6}\right.$, TMS): $\delta 3.53$ (s, 2H, H-11), 6.41 (d, J = $7.92 \mathrm{~Hz}, 1 \mathrm{H}, \mathrm{H}-14), 6.89(\mathrm{t}, J=7.36 \mathrm{~Hz}, 1 \mathrm{H}, \mathrm{H}-$ 16), 7.04-7.09 (m, 2H, H-15,22), 7.22 (d, J = 7.54 Hz, 1H, H-17), 7.25 (d, J = 8.28 Hz, $1 \mathrm{H}$, $\mathrm{H}-8), 7.42$ (d, J = 8.12 Hz, 2H, H-21,23), 8.05 (d, J = 8.28 Hz, 1H, H-7), 8.48 (s, 1H, H-5), 9.10 (bs, $1 \mathrm{H}, \mathrm{H}-18$ ). ${ }^{13} \mathrm{C}$ NMR (100 MHz, DMSO-d, TMS): $\delta 32.53$ (C-11), 93.14 (C-6), 116.25 (C-16), 118.23 (C-10), 120.57 (C-14), 123.74 (C-8), 124.19 (C-22), 126.58 (C-15), 127.05 (C-12), 127.33 (C-21,23), 129.39 (C-20,24), 131.14 (C-17), 137.42 (C-19), 138.87 (C-5), 141.81 (C-13), 144.27 (C-7), 148.62 (C-9), 159.53 (C-4), 164.47 (C-2). Anal. Calcd. for $\mathrm{C}_{21} \mathrm{H}_{13} \mathrm{Cl}_{2} \mathrm{IN}_{2} \mathrm{O}_{2}$ : C, 48.21; $\mathrm{H}, 2.50 ; \mathrm{N}, 5.35$. Found: $\mathrm{C}, 48.25 ; \mathrm{H}, 2.49 ; \mathrm{N}, 5.33$.

\section{General procedure for 1,3,4-oxadiazolyl-quinazolin-4(3H)-ones (9a-f)}

A mixture of benzoxazinones $8 \mathbf{a}-\mathbf{c}(2.5 \mathrm{mmol})$ and 5-substituted phenyl-1,3,4-oxadiazol-2amines $4 \mathbf{a}, \mathbf{b}(2.5 \mathrm{mmol})$ in $10 \mathrm{ml}$ glacial acetic acid was refluxed under anhydrous condition for 4-6 h. After cooling it was poured into crushed ice. The solid separated out was filtered, thoroughly washed with cold distilled water, dried, and recrystallized from ethanol. 


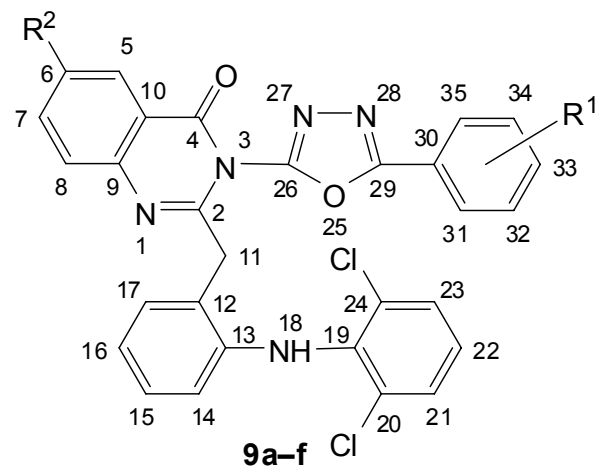

Fig. 4. Numbering of 1,3,4-Oxadiazolyl-quinazolin-4(3H)-ones 9a-f

3-[5-(2-Chlorophenyl)-1,3,4-oxadiazol-2-yl]-2-\{2-[(2,6-dichlorophenyl)amino]benzyl\}quinazolin-4(3H)-one (9a)

Yield: 63\%, mp: 233-235 C. IR (KBr), v, cm ${ }^{-1}: 746$ (C-Cl), 1318 (C-N), 1263, 1037 (C-O-C oxadiazole), 1609 ( $\mathrm{C}=\mathrm{N}$ quinazolinone), $1650 \quad(\mathrm{C}=\mathrm{N}$ oxadiazole), $1678 \quad(\mathrm{C}=\mathrm{O}$ quinazolinone), 2926, $2853\left(\mathrm{CH}_{2}\right), 3445(\mathrm{NH}) .{ }^{1} \mathrm{H}$ NMR (400 MHz, DMSO-d 6 , TMS): $\delta 3.52$ (s, 2H, H-11), 6.41 (d, J = 7.96 Hz, 1H, H-14), 6.89 (t, J=7.4 Hz, 1H, H-16), 7.03-7.09 (m, 2H, H-15,22), 7.22 (d, J = 7.54 Hz, 1H, H-17), $7.41(\mathrm{~d}, J=8.08 \mathrm{~Hz}, 2 \mathrm{H}, \mathrm{H}-21,23), 7.47$ (t, J $=7.68 \mathrm{~Hz}, 1 \mathrm{H}, \mathrm{H}-6), 7.50(\mathrm{t}, J=7.56 \mathrm{~Hz}, 1 \mathrm{H}, \mathrm{H}-33), 7.57(\mathrm{t}, J=7.4 \mathrm{~Hz}, 1 \mathrm{H}, \mathrm{H}-34), 7.61$ (d, $J=8.16 \mathrm{~Hz}, 1 \mathrm{H}, \mathrm{H}-8), 7.67$ (d, $J=7.84 \mathrm{~Hz}, 1 \mathrm{H}, \mathrm{H}-32), 7.71(\mathrm{~d}, J=7.52 \mathrm{~Hz}, 1 \mathrm{H}, \mathrm{H}-35)$, $7.76(\mathrm{t}, J=7.84 \mathrm{~Hz}, 1 \mathrm{H}, \mathrm{H}-7), 8.10(\mathrm{~d}, J=7.76 \mathrm{~Hz}, 1 \mathrm{H}, \mathrm{H}-5), 9.09$ (bs, $1 \mathrm{H}, \mathrm{H}-18) .{ }^{13} \mathrm{C}$ NMR (100 MHz, DMSO-d $d_{6}$ TMS): $\delta 32.54$ (C-11), 116.32 (C-16), 120.41 (C-14), 120.77 (C-10), 122.54 (C-8), 124.31 (C-22), 126.54 (C-15), 127.21 (C-12), 127.39 (C-21,23), 127.49 (C34), 127.58 (C-6), 128.12 (C-35), 128.55 (C-32), 128.76 (C-5), 129.35 (C-20,24), 129.81 (C-33), 131.17 (C-17), 131.45 (C-31), 133.66 (C-7), 135.24 (C-30), 137.32 (C-19), 141.68 (C-13), 147.15 (C-9), 156.72 (C-26), 160.74 (C-4), 163.27 (C-2), 164.65 (C-29). Anal. Calcd. for $\mathrm{C}_{29} \mathrm{H}_{18} \mathrm{Cl}_{3} \mathrm{~N}_{5} \mathrm{O}_{2}$ : C, 60.59; $\mathrm{H}, 3.16 ; \mathrm{N}, 12.18$. Found: $\mathrm{C}, 60.54 ; \mathrm{H}, 3.11 ; \mathrm{N}, 12.21$.

6-Bromo-3-[5-(2-chlorophenyl)-1,3, 4-oxadiazol-2-yl]-2-\{2-[(2, 6-dichlorophenyl)amino]benzyl\}quinazolin-4(3H)-one (9b)

Yield: $55 \%, \mathrm{mp}: 225-228^{\circ} \mathrm{C}$. IR (KBr), v, cm ${ }^{-1}: 570$ (C-Br), 745 (C-Cl), 1311 (C-N), 1260, 1035 (C-O-C oxadiazole), 1611 ( $\mathrm{C}=\mathrm{N}$ quinazolinone), 1653 ( $\mathrm{C}=\mathrm{N}$ oxadiazole), 1675 (C=O quinazolinone), 2925, $2851\left(\mathrm{CH}_{2}\right), 3447(\mathrm{NH}) .{ }^{1} \mathrm{H}$ NMR (400 MHz, DMSO-d 6 , TMS): $\delta 3.53$ (s, 2H, H-11), 6.39 (d, J = 8 Hz, 1H, H-14), 6.89 (t, J = 7.44 Hz, 1H, H-16), 7.03-7.09 (m, $2 \mathrm{H}, \mathrm{H}-15,22), 7.21$ (d, J = 7.58 Hz, 1H, H-17), 7.42 (d, J = 8.16 Hz, 2H, H-21,23), 7.51 (t, J $=7.52 \mathrm{~Hz}, 1 \mathrm{H}, \mathrm{H}-33), 7.55(\mathrm{t}, J=7.36 \mathrm{~Hz}, 1 \mathrm{H}, \mathrm{H}-34), 7.61(\mathrm{~d}, J=8.24 \mathrm{~Hz}, 1 \mathrm{H}, \mathrm{H}-8), 7.66$ $(\mathrm{d}, J=7.8 \mathrm{~Hz}, 1 \mathrm{H}, \mathrm{H}-32), 7.70(\mathrm{~d}, J=7.48 \mathrm{~Hz}, 1 \mathrm{H}, \mathrm{H}-35), 8.08(\mathrm{~d}, J=8.24 \mathrm{~Hz}, 1 \mathrm{H}, \mathrm{H}-7)$, 8.12 (s, 1H, H-5), 9.08 (bs, $1 \mathrm{H}, \mathrm{H}-18) .{ }^{13} \mathrm{C}$ NMR (100 MHz, DMSO-d, TMS): $\delta 32.65$ (C11), 116.24 (C-16), 120.53 (C-14), 123.13 (C-10), 124.24 (C-22), 124.58 (C-8), 126.47 (C15), 127.18 (C-12), 127.37 (C-21,23), 127.48 (C-34), 127.66 (C-6), 128.15 (C-35), 128.72 (C-32), 129.34 (C-20,24), 129.79 (C-33), 131.16 (C-17), 131.47 (C-31), 132.25 (C-5), 135.21 (C-30), 136.37 (C-7), 137.36 (C-19), 141.77 (C-13), 146.21 (C-9), 156.64 (C-26), 160.59 (C-4), 163.38 (C-2), 164.57 (C-29). Anal. Calcd. for $\mathrm{C}_{29} \mathrm{H}_{17} \mathrm{BrCl}_{3} \mathrm{~N}_{5} \mathrm{O}_{2}$ : C, 53.28; $\mathrm{H}$, 2.62; N, 10.71. Found: C, 53.19; H, 2.65; N, 10.75 . 
3-[5-(2-Chlorophenyl)-1,3,4-oxadiazol-2-yl]-2-\{2-[(2,6-dichlorophenyl)amino]benzyl\}6-iodoquinazolin-4(3H)-one (9c)

Yield: 66\%, mp: $241-244^{\circ} \mathrm{C}$. IR (KBr), v, cm ${ }^{-1}: 618$ (C-I), 740 (C-Cl), 1317 (C-N), 1262, 1042 (C-O-C oxadiazole), 1608 ( $\mathrm{C}=\mathrm{N}$ quinazolinone), 1655 ( $\mathrm{C}=\mathrm{N}$ oxadiazole), 1684 ( $\mathrm{C}=\mathrm{O}$ quinazolinone), 2928, $2854\left(\mathrm{CH}_{2}\right), 3449(\mathrm{NH}) .{ }^{1} \mathrm{H}$ NMR (400 MHz, DMSO- $\left.d_{6}, \mathrm{TMS}\right): \delta 3.52$ (s, 2H, H-11), 6.40 (d, J = 7.92 Hz, 1H, H-14), 6.89 (t, J = 7.36 Hz, 1H, H-16), 7.04-7.10 (m, 2H, H-15,22), 7.21 (d, J = 7.5 Hz, 1H, H-17), 7.25 (d, J = 8.28 Hz, 1H, H-8), 7.41 (d, J $=8.12 \mathrm{~Hz}, 2 \mathrm{H}, \mathrm{H}-21,23), 7.48(\mathrm{t}, J=7.56 \mathrm{~Hz}, 1 \mathrm{H}, \mathrm{H}-33), 7.55(\mathrm{t}, J=7.9 \mathrm{~Hz}, 1 \mathrm{H}, \mathrm{H}-36)$, 7.64 (d, $J=7.84 \mathrm{~Hz}, 1 \mathrm{H}, \mathrm{H}-32), 7.69$ (d, $J=7.48 \mathrm{~Hz}, 1 \mathrm{H}, \mathrm{H}-35), 7.93(\mathrm{~d}, J=8.28 \mathrm{~Hz}, 1 \mathrm{H}$, $\mathrm{H}-7$ ), 8.28 (s, 1H, H-5), 9.11 (bs, $1 \mathrm{H}, \mathrm{H}-18) .{ }^{13} \mathrm{C}$ NMR (100 MHz, DMSO- $\left.d_{6}, \mathrm{TMS}\right): \delta 32.95$ (C-11), 93.15 (C-6), 116.25 (C-16), 120.44 (C-14), 122.56 (C-10), 124.02 (C-8), 124.23 (C22), 126.50 (C-15), 127.13 (C-12), 127.34 (C-21,23), 127.57 (C-34), 128.14 (C-35), 128.71 (C-32), 129.47 (C-20,24), 129.82 (C-33), 131.30 (C-17), 131.46 (C-31), 135.19 (C-30), 136.24 (C-5), 137.35 (C-19), 141.78 (C-13), 142.43 (C-7), 146.11 (C-9), 156.69 (C-26), 160.88 (C-4), 163.35 (C-2), 164.61 (C-29). Anal. Calcd. for $\mathrm{C}_{29} \mathrm{H}_{17} \mathrm{Cl}_{3} \mathrm{IN}_{5} \mathrm{O}_{2}: \mathrm{C}, 49.71 ; \mathrm{H}$, $2.45 ;$ N, 9.99. Found: C, 49.66; H, 2.51; N, 10.02 .

\section{3-[5-(4-Chlorophenyl)-1,3,4-oxadiazol-2-yl]-2-\{2-[(2,6-dichlorophenyl)amino]benzyl\}-} quinazolin-4(3H)-one (9d)

Yield: 65\%, mp: $260-265^{\circ} \mathrm{C}$. IR (KBr), v, cm ${ }^{-1}: 748$ (C-Cl), 1315 (C-N), 1264, 1038 (C-O-C oxadiazole), $1612 \quad(\mathrm{C}=\mathrm{N}$ quinazolinone $), 1648 \quad(\mathrm{C}=\mathrm{N}$ oxadiazole $), 1675 \quad(\mathrm{C}=\mathrm{O}$ quinazolinone), 2923, $2848\left(\mathrm{CH}_{2}\right), 3442(\mathrm{NH}) .{ }^{1} \mathrm{H}$ NMR (400 MHz, DMSO-d 6 , TMS): $\delta 3.51$ (s, 2H, H-11), 6.38 (d, J = 8.04 Hz, 1H, H-14), 6.89 (t, J = 7.48 Hz, 1H, H-16), 7.03-7.10 (m, 2H, H-15,22), 7.22 (d, J = 7.62 Hz, 1H, H-17), 7.43 (d, J = 8.12 Hz, 2H, H-21,23), 7.49 $(\mathrm{t}, J=7.6 \mathrm{~Hz}, 1 \mathrm{H}, \mathrm{H}-6), 7.61(\mathrm{~d}, J=8.08 \mathrm{~Hz}, 1 \mathrm{H}, \mathrm{H}-8), 7.65(\mathrm{~d}, J=8.24 \mathrm{~Hz}, 2 \mathrm{H}, \mathrm{H}-32,34)$, $7.70(\mathrm{~d}, J=8.24 \mathrm{~Hz}, 2 \mathrm{H}, \mathrm{H}-31,35), 7.74(\mathrm{t}, J=7.8 \mathrm{~Hz}, 1 \mathrm{H}, \mathrm{H}-7), 8.11(\mathrm{~d}, J=7.72 \mathrm{~Hz}, 1 \mathrm{H}$, $\mathrm{H}-5), 9.07$ (bs, $1 \mathrm{H}, \mathrm{H}-18) .{ }^{13} \mathrm{C}$ NMR (100 MHz, DMSO-d 6 , TMS): $\delta 32.51$ (C-11), 116.30 (C-16), 120.43 (C-14), 120.75 (C-10), 122.52 (C-8), 123.32 (C-30), 124.33 (C-22), 126.57 (C-15), 127.23 (C-12), 127.41 (C-21,23), 127.60 (C-6), 128.78 (C-5), 129.37 (C-20,24), 131.20 (C-17), 131.82 (C-31,35), 133.21 (C-32,34), 133.68 (C-7), 135.43 (C-33), 137.36 (C-19), 141.70 (C-13), 147.17 (C-9), 156.62 (C-26), 160.78 (C-4), 163.37 (C-2), 164.67 (C29). Anal. Calcd. for $\mathrm{C}_{29} \mathrm{H}_{18} \mathrm{Cl}_{3} \mathrm{~N}_{5} \mathrm{O}_{2}$ : C, 60.59; $\mathrm{H}, 3.16 ; \mathrm{N}, 12.18$. Found: $\mathrm{C}, 60.51 ; \mathrm{H}, 3.19$; $\mathrm{N}, 12.15$.

\section{6-Bromo-3-[5-(4-chlorophenyl)-1,3,4-oxadiazol-2-yl]-2-\{2-[(2, 6-dichlorophenyl)amino]- benzyl\}quinazolin-4(3H)-one (9e)}

Yield: $61 \%, \mathrm{mp}: 254-258^{\circ} \mathrm{C}$. IR (KBr), v, cm ${ }^{-1}: 568$ (C-Br), $743(\mathrm{C}-\mathrm{Cl}), 1316(\mathrm{C}-\mathrm{N}), 1258$, 1032 (C-O-C oxadiazole), 1613 (C=N quinazolinone), 1650 ( $\mathrm{C}=\mathrm{N}$ oxadiazole), 1674 ( $\mathrm{C}=\mathrm{O}$ quinazolinone), 2922, $2849\left(\mathrm{CH}_{2}\right), 3444(\mathrm{NH}) .{ }^{1} \mathrm{H}$ NMR (400 MHz, DMSO-d 6 , TMS): $\delta 3.52$ (s, 2H, H-11), $6.41(\mathrm{~d}, J=8 \mathrm{~Hz}, 1 \mathrm{H}, \mathrm{H}-14), 6.90(\mathrm{t}, J=7.44 \mathrm{~Hz}, 1 \mathrm{H}, \mathrm{H}-16), 7.04-7.10(\mathrm{~m}$, $2 \mathrm{H}, \mathrm{H}-15,22), 7.20$ (d, J = 7.58 Hz, 1H, H-17), 7.42 (d, J = 8.08 Hz, 2H, H-21,23), 7.60 (d, $J=8.32 \mathrm{~Hz}, 1 \mathrm{H}, \mathrm{H}-8), 7.64(\mathrm{~d}, J=8.2 \mathrm{~Hz}, 2 \mathrm{H}, \mathrm{H}-32,34), 7.69$ (d, $J=8.2 \mathrm{~Hz}, 2 \mathrm{H}, \mathrm{H}-31,35)$, 8.01 (d, $J=8.32 \mathrm{~Hz}, 1 \mathrm{H}, \mathrm{H}-7), 8.09$ (s, $1 \mathrm{H}, \mathrm{H}-5), 9.08$ (bs, $1 \mathrm{H}, \mathrm{H}-18) .{ }^{13} \mathrm{C}$ NMR $(100 \mathrm{MHz}$, DMSO- $d_{6}$, TMS): $\delta 32.57$ (C-11), 116.28 (C-16), 120.56 (C-14), 123.16 (C-10), 123.33 (C30), 124.27 (C-22), 124.56 (C-8), 126.48 (C-15), 127.22 (C-12), 127.39 (C-21,23), 127.67 (C-6), 129.35 (C-20,24), 131.18 (C-17), 131.79 (C-31,35), 132.24 (C-5), 133.24 (C-32,34), 135.39 (C-33), 136.35 (C-7), 137.38 (C-19), 141.76 (C-13), 146.23 (C-9), 156.58 (C-26), 
160.61 (C-4), 163.41 (C-2), 164.54 (C-29). Anal. Calcd. for $\mathrm{C}_{29} \mathrm{H}_{17} \mathrm{BrCl}_{3} \mathrm{~N}_{5} \mathrm{O}_{2}$ : C, 53.28; $\mathrm{H}$, 2.62; N, 10.71. Found: C, 53.35; H, 2.66; N, 10.58.

3-[5-(4-Chlorophenyl)-1,3,4-oxadiazol-2-yl]-2-\{2-[(2, 6-dichlorophenyl)amino]benzyl\}6-iodoquinazolin-4(3H)-one (9f)

Yield: 68\%, mp: 273-277 C. IR (KBr), v, cm ${ }^{-1}: 615$ (C-I), 746 (C-Cl), 1315 (C-N), 1260, 1045 (C-O-C oxadiazole), 1612 ( $\mathrm{C}=\mathrm{N}$ quinazolinone), 1651 ( $\mathrm{C}=\mathrm{N}$ oxadiazole), 1680 ( $\mathrm{C}=\mathrm{O}$ quinazolinone), 2927, $2852\left(\mathrm{CH}_{2}\right), 3450(\mathrm{NH}) .{ }^{1} \mathrm{H}$ NMR (400 MHz, DMSO-d 6 , TMS): $\delta 3.51$ (s, 2H, H-11), 6.41 (d, J = 7.92 Hz, 1H, H-14), 6.88 (t, J = 7.36 Hz, 1H, H-16), 7.05-7.08 $(\mathrm{m}, 2 \mathrm{H}, \mathrm{H}-15,22), 7.21$ (d, J = 7.54 Hz, 1H, H-17), 7.25 (d, J = 8.36 Hz, 1H, H-8), 7.41 (d, J $=8.12 \mathrm{~Hz}, 2 \mathrm{H}, \mathrm{H}-21,23), 7.62(\mathrm{~d}, J=8.28 \mathrm{~Hz}, 2 \mathrm{H}, \mathrm{H}-32,34), 7.68(\mathrm{~d}, J=8.28 \mathrm{~Hz}, 2 \mathrm{H}, \mathrm{H}-$ 31,35), 7.95 (d, J = 8.36 Hz, 1H, H-7), 8.28 (s, 1H, H-5), 9.11 (bs, $1 \mathrm{H}, \mathrm{H}-18) .{ }^{13} \mathrm{C}$ NMR (100 MHz, DMSO-d $d_{6}$ TMS): $\delta 33.05$ (C-11), 93.18 (C-6), 116.29 (C-16), 120.42 (C-14), 122.58 (C-10), 123.30 (C-30), 124.08 (C-8), 124.25 (C-22), 126.52 (C-15), 127.16 (C-12), 127.35 (C-21,23), 129.46 (C-20,24), 131.32 (C-17), 131.77 (C-31,35), 133.25 (C-32,34), 135.41 (C-33), 136.23 (C-5), 137.34 (C-19), 141.76 (C-13), 142.45 (C-7), 146.13 (C-9), 156.65 (C-26), 160.89 (C-4), 163.37 (C-2), 164.62 (C-29). Anal. Calcd. for $\mathrm{C}_{29} \mathrm{H}_{17} \mathrm{Cl}_{3} \mathrm{IN}_{5} \mathrm{O}_{2}$ : C, 49.71; H, 2.45; N, 9.99. Found: C, 49.65; H, 2.47; N, 10.04.

\section{General procedure for 1,3,4-oxadiazolyl-quinazolin-4(3H)-ones (10a-f)}

A mixture of benzoxazinones $8 \mathrm{a}-\mathrm{c}(2.5 \mathrm{mmol})$ and 4-(5-substituted phenyl-1,3,4oxadiazol-2-yl)benzenamines $\mathbf{5 a}, \mathbf{b}(2.5 \mathrm{mmol}) 10 \mathrm{ml}$ in pyridine was refluxed on an oil bath for 6-8 $\mathrm{h}$. After completion of the reaction, the oily mass was slowly poured onto crushed ice cold water containing $5 \mathrm{ml}$ concentrated $\mathrm{HCl}$ with continues stirring. The product obtained was filtered and washed several times with cold water, dried and recrystallized from ethanol.

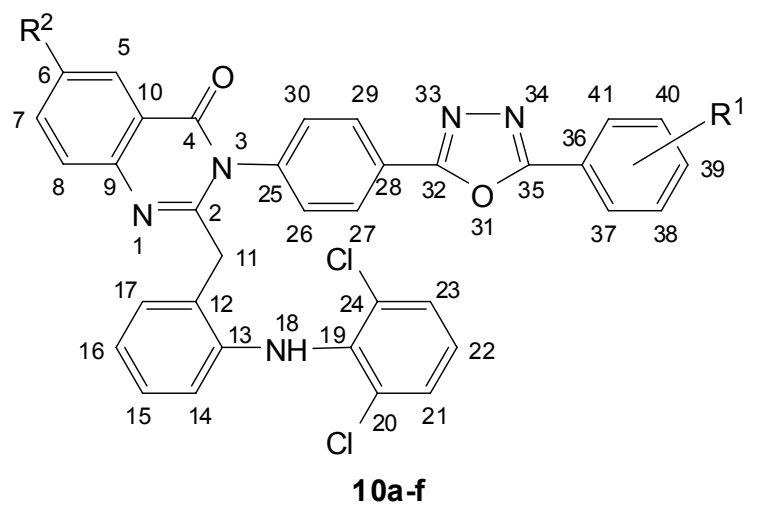

Fig. 5. Numbering of 1,3,4-Oxadiazolyl-quinazolin-4(3H)-ones 10a-f

3-\{4-[5-(2-Chlorophenyl)-1,3,4-oxadiazol-2-yl]phenyl\}-2-\{2-[(2,6-dichlorophenyl)amino]benzyl\}quinazolin-4(3H)-one (10a)

Yield: 62\%, mp: $228-231^{\circ} \mathrm{C}$. IR (KBr), v, $\mathrm{cm}^{-1}: 740$ (C-Cl), 1316 (C-N), 1265, 1041 (C-O-C oxadiazole), $1610 \quad(\mathrm{C}=\mathrm{N}$ quinazolinone $), 1656 \quad(\mathrm{C}=\mathrm{N}$ oxadiazole), $1676 \quad(\mathrm{C}=\mathrm{O}$ quinazolinone), 2925, $2852\left(\mathrm{CH}_{2}\right), 3446(\mathrm{NH}) .{ }^{1} \mathrm{H}$ NMR (400 MHz, DMSO-d 6 , TMS): $\delta 3.51$ (s, 2H, H-11), 6.40 (d, J = $8 \mathrm{~Hz}, 1 \mathrm{H}, \mathrm{H}-14), 6.89$ (t, J=7.4 Hz, 1H, H-16), 7.03-7.09 (m, 
$2 \mathrm{H}, \mathrm{H}-15,22), 7.22$ (d, J = 7.58 Hz, 1H, H-17), 7.42 (d, J=8.16 Hz, 2H, H-21,23), 7.45 (d, $J=8.4 \mathrm{~Hz}, 2 \mathrm{H}, \mathrm{H}-26,30), 7.47-7.55(\mathrm{~m}, 4 \mathrm{H}, \mathrm{Ar}-\mathrm{H}), 7.57$ (t, J = 7.36 Hz, 1H, H-40), 7.62 (d, $J=8.16 \mathrm{~Hz}, 1 \mathrm{H}, \mathrm{H}-8), 7.66(\mathrm{~d}, J=7.8 \mathrm{~Hz}, 1 \mathrm{H}, \mathrm{H}-38), 7.71(\mathrm{~d}, J=7.48 \mathrm{~Hz}, 1 \mathrm{H}, \mathrm{H}-41), 7.75$ $(\mathrm{t}, J=7.84 \mathrm{~Hz}, 1 \mathrm{H}, \mathrm{H}-7), 8.07$ (d, J = 7.74 Hz, 1H, H-5), 9.11 (bs, 1H, H-18). ${ }^{13} \mathrm{C}$ NMR (100 MHz, DMSO-d $\left.d_{6}, \mathrm{TMS}\right): \delta 32.48$ (C-11), 116.27 (C-16), 120.43 (C-14), 120.81 (C-10), 121.47 (C-28), 121.82 (C-26,30), 122.58 (C-8), 124.34 (C-22), 126.57 (C-15), 127.23 (C12), 127.40 (C-21,23), 127.51 (C-40), 127.61 (C-6), 127.72 (C-27,29), 128.14 (C-41), 128.67 (C-38), 128.78 (C-5), 129.33 (C-20,24), 129.78 (C-39), 131.18 (C-17), 131.43 (C37), 132.64 (C-25), 133.62 (C-7), 135.22 (C-36), 137.34 (C-19), 141.67 (C-13), 147.12 (C9), 160.73 (C-4), 163.26 (C-2), 164.64 (C-32, 35). Anal. Calcd. for $\mathrm{C}_{35} \mathrm{H}_{22} \mathrm{Cl}_{3} \mathrm{~N}_{5} \mathrm{O}_{2}$ : C, 64.58; H, 3.41; N, 10.76. Found: C, 64.51; H, 3.46; N, 10.71.

\section{6-Bromo-3-\{4-[5-(2-chlorophenyl)-1,3,4-oxadiazol-2-yl]phenyl\}-2-\{2-[(2, 6-dichlorophenyl)- amino]benzyl\}quinazolin-4(3H)-one (10b)}

Yield: $64 \%, \mathrm{mp}: 213-217^{\circ} \mathrm{C}$. IR (KBr), v, cm ${ }^{-1}: 572$ (C-Br), $743(\mathrm{C}-\mathrm{Cl}), 1312(\mathrm{C}-\mathrm{N}), 1268$, 1048 (C-O-C oxadiazole), 1609 ( $\mathrm{C}=\mathrm{N}$ quinazolinone), 1653 ( $\mathrm{C}=\mathrm{N}$ oxadiazole), 1681 ( $\mathrm{C}=\mathrm{O}$ quinazolinone), 2921, $2847\left(\mathrm{CH}_{2}\right), 3442(\mathrm{NH}) .{ }^{1} \mathrm{H}$ NMR (400 MHz, DMSO-d 6 , TMS): $\delta 3.53$ (s, 2H, H-11), 6.42 (d, J = 7.96 Hz, 1H, H-14), 6.91 (t, $J=7.44 \mathrm{~Hz}, 1 \mathrm{H}, \mathrm{H}-16), 7.03-7.10$ (m, 2H, H-15,22), 7.23 (d, J = 7.58 Hz, 1H, H-17), 7.42 (d, J = 8.12 Hz, 2H, H-21,23), 7.45 (d, J = 8.36 Hz, 2H, H-26,30), 7.48 (t, J = 7.56 Hz, 1H, H-39), $7.54(\mathrm{t}, J=7.4 \mathrm{~Hz}, 1 \mathrm{H}, \mathrm{H}-$ 40), 7.57 (d, J = 8.36 Hz, 2H, H-27,29), 7.61 (d, J = 8.4 Hz, 1H, H-8), 7.65 (d, J = 7.8 Hz, $1 \mathrm{H}, \mathrm{H}-38), 7.72$ (d, J=7.48 Hz, 1H, H-41), 8.05 (d, J = 8.4 Hz, 1H, H-7), $8.12(\mathrm{~s}, 1 \mathrm{H}, \mathrm{H}-5)$, 9.11 (bs, $1 \mathrm{H}, \mathrm{H}-18) .{ }^{13} \mathrm{C}$ NMR (100 MHz, DMSO-d, TMS): $\delta 32.63$ (C-11), 116.27 (C-16), 120.55 (C-14), 121.52 (C-28), 121.78 (C-26,30), 123.12 (C-10), 124.26 (C-22), 124.62 (C8), 126.50 (C-15), 127.17 (C-12), 127.38 (C-21,23), 127.53 (C-40), 127.64 (C-6), 127.73 (C-27,29), 128.17 (C-41), 128.76 (C-38), 129.37 (C-20,24), 129.82 (C-39), 131.20 (C-17), 131.45 (C-37), 132.27 (C-5), 132.64 (C-25), 135.23 (C-36), 136.35 (C-7), 137.32 (C-19), 141.74 (C-13), 146.23 (C-9), 160.60 (C-4), 163.28 (C-2), 164.59 (C-32,35). Anal. Calcd. for $\mathrm{C}_{35} \mathrm{H}_{21} \mathrm{BrCl}_{3} \mathrm{~N}_{5} \mathrm{O}_{2}$ : C, 57.60; H, 2.90; N, 9.60. Found: C, 57.52; H, 2.94; N, 9.63.

\section{3-\{4-[5-(2-Chlorophenyl)-1,3,4-oxadiazol-2-yl]phenyl\}-2-\{2-[(2, 6-dichlorophenyl)amino]- benzyl\}-6-iodoquinazolin-4(3H)-one (10c)}

Yield: $60 \%, \mathrm{mp}: 235-238^{\circ} \mathrm{C} . \mathrm{IR}(\mathrm{KBr}), \mathrm{v}, \mathrm{cm}^{-1}: 620$ (C-I), 748 (C-Cl), 1312 (C-N), 1270, 1051 (C-O-C oxadiazole), 1611 ( $\mathrm{C}=\mathrm{N}$ quinazolinone), 1649 ( $\mathrm{C}=\mathrm{N}$ oxadiazole), 1678 ( $\mathrm{C}=\mathrm{O}$ quinazolinone), 2928, $2855\left(\mathrm{CH}_{2}\right), 3448(\mathrm{NH}) .{ }^{1} \mathrm{H}$ NMR (400 MHz, DMSO-d 6 , TMS): $\delta 3.52$ (s, 2H, H-11), $6.41(\mathrm{~d}, J=8 \mathrm{~Hz}, 1 \mathrm{H}, \mathrm{H}-14), 6.88(\mathrm{t}, J=7.44 \mathrm{~Hz}, 1 \mathrm{H}, \mathrm{H}-16), 7.05-7.11(\mathrm{~m}$, $2 \mathrm{H}, \mathrm{H}-15,22), 7.23(\mathrm{~d}, J=7.54 \mathrm{~Hz}, 1 \mathrm{H}, \mathrm{H}-17), 7.26(\mathrm{~d}, J=8.32 \mathrm{~Hz}, 1 \mathrm{H}, \mathrm{H}-8), 7.43(\mathrm{~d}, J=$ $8.04 \mathrm{~Hz}, 2 \mathrm{H}, \mathrm{H}-21,23), 7.46(\mathrm{~d}, J=8.44 \mathrm{~Hz}, 2 \mathrm{H}, \mathrm{H}-26,30), 7.49$ (t, J=7.6 Hz, 1H, H-39), $7.53(\mathrm{~d}, J=8.44 \mathrm{~Hz}, 2 \mathrm{H}, \mathrm{H}-27,29), 7.56(\mathrm{t}, J=7.42 \mathrm{~Hz}, 1 \mathrm{H}, \mathrm{H}-40), 7.63$ (d, J = $7.88 \mathrm{~Hz}$, $1 \mathrm{H}, \mathrm{H}-38$ ), 7.69 (d, J = 7.56 Hz, 1H, H-41), 7.93 (d, J = 8.32 Hz, 1H, H-7), $8.28(\mathrm{~s}, 1 \mathrm{H}, \mathrm{H}-$ 5), 9.09 (bs, $1 \mathrm{H}, \mathrm{H}-18) .{ }^{13} \mathrm{C}$ NMR (100 MHz, DMSO-d 6 , TMS): $\delta 32.90$ (C-11), 93.21 (C-6), 116.29 (C-16), 120.48 (C-14), 121.49 (C-28), 121.81 (C-26,30), 122.55 (C-10), 124.05 (C8), 124.25 (C-22), 126.49 (C-15), 127.15 (C-12), 127.38 (C-21,23), 127.49 (C-40), 127.68 (C-27,29), 128.12 (C-41), 128.68 (C-38), 129.44 (C-20,24), 129.74 (C-39), 131.29 (C-17), 131.40 (C-37), 132.58 (C-25), 135.18 (C-36), 136.21 (C-5), 137.37 (C-19), 141.75 (C-13), 142.46 (C-7), 146.09 (C-9), 160.95 (C-4), 163.22 (C-2), 164.63 (C-32,35). Anal. Calcd. for $\mathrm{C}_{35} \mathrm{H}_{21} \mathrm{Cl}_{3} \mathrm{IN}_{5} \mathrm{O}_{2}: \mathrm{C}, 54.11 ; \mathrm{H}, 2.72 ; \mathrm{N}, 9.02$. Found: $\mathrm{C}, 54.18 ; \mathrm{H}, 2.76 ; \mathrm{N}, 8.95$. 
3-\{4-[5-(4-Chlorophenyl)-1,3,4-oxadiazol-2-yl]phenyl\}-2-\{2-[(2,6-dichlorophenyl)amino]benzyl\}quinazolin-4(3H)-one (10d)

Yield: 59\%, mp: $245-248^{\circ} \mathrm{C}$. IR (KBr), v, cm ${ }^{-1}: 748$ (C-Cl), 1318 (C-N), 1275, 1045 (C-O-C oxadiazole), 1613 ( $\mathrm{C}=\mathrm{N}$ quinazolinone), $1658 \quad(\mathrm{C}=\mathrm{N}$ oxadiazole), $1680 \quad(\mathrm{C}=\mathrm{O}$ quinazolinone), 2925, $2852\left(\mathrm{CH}_{2}\right), 3451(\mathrm{NH}) .{ }^{1} \mathrm{H}$ NMR (400 MHz, DMSO-d 6 , TMS): $\delta 3.52$ (s, 2H, H-11), 6.40 (d, J = 7.88 Hz, 1H, H-14), 6.89 (t, $J=7.36 \mathrm{~Hz}, 1 \mathrm{H}, \mathrm{H}-16), 7.04-7.09$ (m, 2H, H-15,22), $7.21(\mathrm{~d}, J=7.5 \mathrm{~Hz}, 1 \mathrm{H}, \mathrm{H}-17), 7.42$ (d, J = 8.08 Hz, 2H, H-21,23), 7.45 $(\mathrm{d}, J=8.36 \mathrm{~Hz}, 2 \mathrm{H}, \mathrm{H}-26,30), 7.49(\mathrm{t}, J=7.6 \mathrm{~Hz}, 1 \mathrm{H}, \mathrm{H}-6), 7.56(\mathrm{~d}, J=8.36 \mathrm{~Hz}, 2 \mathrm{H}, \mathrm{H}-$ 27,29), 7.61 (d, J = 8.12 Hz, 1H, H-8), 7.65 (d, J = 8.28 Hz, 2H, H-37,41), 7.68 (d, J = 8.28 $\mathrm{Hz}, 2 \mathrm{H}, \mathrm{H}-38,40), 7.75$ (t, J = 7.8 Hz, 1H, H-7), 8.09 (d, J = 7.68 Hz, 1H, H-5), 9.10 (bs, $1 \mathrm{H}, \mathrm{H}-18) .{ }^{13} \mathrm{C}$ NMR (100 MHz, DMSO- $\left.d_{6}, \mathrm{TMS}\right): \delta 32.61$ (C-11), 116.30 (C-16), 120.45 (C-14), 120.75 (C-10), 121.52 (C-28), 121.78 (C-26,30), 122.57 (C-8), 123.28 (C-36), 124.32 (C-22), 126.51 (C-15), 127.19 (C-12), 127.37 (C-21,23), 127.56 (C-6), 127.68 (C27,29), 128.74 (C-5), 129.32 (C-20,24), 131.15 (C-17), 131.83 (C-37,41), 132.59 (C-25), 133.19 (C-38,40), 133.64 (C-7), 135.42 (C-39), 137.34 (C-19), 141.65 (C-13), 147.13 (C9), 160.77 (C-4), 163.38 (C-2), 164.61 (C-32,35). Anal. Calcd. for $\mathrm{C}_{35} \mathrm{H}_{22} \mathrm{Cl}_{3} \mathrm{~N}_{5} \mathrm{O}_{2}: \mathrm{C}, 64.58$; $\mathrm{H}, 3.41 ; \mathrm{N}, 10.76$. Found: $\mathrm{C}, 64.53 ; \mathrm{H}, 3.44 ; \mathrm{N}, 10.73$.

6-Bromo-3-\{4-[5-(4-chlorophenyl)-1,3,4-oxadiazol-2-yl]phenyl\}-2-\{2-[(2, 6-dichlorophenyl)amino]benzyl\}quinazolin-4(3H)-one (10e)

Yield: $68 \%, \mathrm{mp}: 221-224^{\circ} \mathrm{C}$. IR (KBr), v, cm ${ }^{-1}: 565$ (C-Br), 747 (C-Cl), 1317 (C-N), 1266, 1044 (C-O-C oxadiazole), 1609 (C=N quinazolinone), 1650 ( $\mathrm{C}=\mathrm{N}$ oxadiazole), 1676 ( $\mathrm{C}=\mathrm{O}$ quinazolinone), 2923, $2852\left(\mathrm{CH}_{2}\right), 3446(\mathrm{NH}) .{ }^{1} \mathrm{H}$ NMR (400 MHz, DMSO-d 6 , TMS): $\delta 3.53$ (s, 2H, H-11), 6.41 (d, J = 7.96 Hz, 1H, H-14), 6.90 (t, J = 7.4 Hz, 1H, H-16), 7.04-7.10 (m, $2 \mathrm{H}, \mathrm{H}-15,22), 7.20$ (d, J = 7.58 Hz, 1H, H-17), 7.42 (d, J = 8.12 Hz, 2H, H-21,23), 7.46 (d, $J=8.4 \mathrm{~Hz}, 2 \mathrm{H}, \mathrm{H}-26,30), 7.56(\mathrm{~d}, J=8.4 \mathrm{~Hz}, 2 \mathrm{H}, \mathrm{H}-27,29), 7.60(\mathrm{~d}, J=8.28 \mathrm{~Hz}, 1 \mathrm{H}, \mathrm{H}-8)$, $7.64(\mathrm{~d}, J=8.24 \mathrm{~Hz}, 2 \mathrm{H}, \mathrm{H}-38,40), 7.69$ (d, J = 8.24 Hz, 2H, H-37,41), 8.07 (d, J = 8.28 $\mathrm{Hz}, 1 \mathrm{H}, \mathrm{H}-7), 8.13(\mathrm{~s}, 1 \mathrm{H}, \mathrm{H}-5), 9.11$ (bs, $1 \mathrm{H}, \mathrm{H}-18) .{ }^{13} \mathrm{C}$ NMR (100 MHz, DMSO-d 6 , TMS): ठ 32.67 (C-11), 116.25 (C-16), 120.56 (C-14), 121.53 (C-28), 121.79 (C-26,30), 123.16 (C10), 123.32 (C-36), 124.27 (C-22), 124.56 (C-8), 126.44 (C-15), 127.15 (C-12), 127.34 (C21,23), 127.62 (C-6), 127.74 (C-27,29), 129.38 (C-20,24), 131.22 (C-17), 131.82 (C37,41), 132.28 (C-5), 132.62 (C-25), 133.22 (C-38,40), 135.41 (C-39), 136.35 (C-7), 137.32 (C-19), 141.73 (C-13), 146.25 (C-9), 160.56 (C-4), 163.37 (C-2), 164.52 (C-32,35). Anal. Calcd. for $\mathrm{C}_{35} \mathrm{H}_{21} \mathrm{BrCl}_{3} \mathrm{~N}_{5} \mathrm{O}_{2}$ : C, 57.60; H, 2.90; N, 9.60. Found: C, 57.53; H, 2.93; N, 9.55 .

3-\{4-[5-(4-Chlorophenyl)-1,3,4-oxadiazol-2-yl]phenyl\}-2-\{2-[(2,6-dichlorophenyl)amino]benzyl\}-6-iodoquinazolin-4(3H)-one (10f)

Yield: 65\%, mp: $257-261^{\circ} \mathrm{C}$. IR (KBr), v, cm ${ }^{-1}: 617$ (C-I), 745 (C-Cl), 1314 (C-N), 1268, 1052 (C-O-C oxadiazole), 1608 ( $\mathrm{C}=\mathrm{N}$ quinazolinone), 1652 ( $\mathrm{C}=\mathrm{N}$ oxadiazole), 1679 ( $\mathrm{C}=\mathrm{O}$ quinazolinone), 2925, $2851\left(\mathrm{CH}_{2}\right), 3443(\mathrm{NH}) .{ }^{1} \mathrm{H}$ NMR (400 MHz, DMSO-d 6 , TMS): $\delta 3.51$ (s, 2H, H-11), $6.39(\mathrm{~d}, J=8 \mathrm{~Hz}, 1 \mathrm{H}, \mathrm{H}-14), 6.88(\mathrm{t}, J=7.48 \mathrm{~Hz}, 1 \mathrm{H}, \mathrm{H}-16), 7.03-7.10(\mathrm{~m}$, $2 \mathrm{H}, \mathrm{H}-15,22), 7.20(\mathrm{~d}, J=7.58 \mathrm{~Hz}, 1 \mathrm{H}, \mathrm{H}-17), 7.24(\mathrm{~d}, J=8.24 \mathrm{~Hz}, 1 \mathrm{H}, \mathrm{H}-8), 7.43(\mathrm{~d}, J=$ $8.16 \mathrm{~Hz}, 2 \mathrm{H}, \mathrm{H}-21,23), 7.47(\mathrm{~d}, J=8.32 \mathrm{~Hz}, 2 \mathrm{H}, \mathrm{H}-26,30), 7.58(\mathrm{~d}, J=8.32 \mathrm{~Hz}, 2 \mathrm{H}, \mathrm{H}-$ 27,29), 7.63 (d, J = 8.2 Hz, 2H, H-38,40), 7.68 (d, J=8.2 Hz, 2H, H-37,41), 7.95 (d, J = $8.24 \mathrm{~Hz}, 1 \mathrm{H}, \mathrm{H}-7), 8.28$ (s, $1 \mathrm{H}, \mathrm{H}-5), 9.12$ (bs, $1 \mathrm{H}, \mathrm{H}-18) .{ }^{13} \mathrm{C}$ NMR (100 MHz, DMSO-d 6 , TMS): $\delta 33.01$ (C-11), 93.20 (C-6), 116.21 (C-16), 120.43 (C-14), 121.48 (C-28), 121.76 
(C-26,30), 122.55 (C-10), 123.34 (C-36), 124.06 (C-8), 124.25 (C-22), 126.48 (C-15), 127.14 (C-12), 127.32 (C-21,23), 127.67 (C-27,29), 129.43 (C-20,24), 131.33 (C-17), 131.82 (C-37,41), 132.63 (C-25), 133.23 (C-38,40), 135.44 (C-39), 136.28 (C-5), 137.34 (C-19), 141.76 (C-13), 142.48 (C-7), 146.15 (C-9), 160.93 (C-4), 163.31 (C-2), 164.59 (C32,35). Anal. Calcd. for $\mathrm{C}_{35} \mathrm{H}_{21} \mathrm{Cl}_{3} \mathrm{IN}_{5} \mathrm{O}_{2}$ : C, 54.11; $\mathrm{H}, 2.72 ; \mathrm{N}, 9.02$. Found: $\mathrm{C}, 54.06 ; \mathrm{H}$, $2.75 ; \mathrm{N}, 9.09$.

\section{General procedure for quinazolin-4(3H)-ones (11a-c)}

A mixture of benzoxazinones $8 \mathbf{a}-\mathbf{c}(5 \mathrm{mmol})$ and glycine $(5 \mathrm{mmol})$ was refluxed in $20 \mathrm{ml}$ butanol for 6-8 h. After completion of the reaction, it was concentrated and after cooling, water was added and solid thus obtained was filtered off and recrystallized from ethanol.

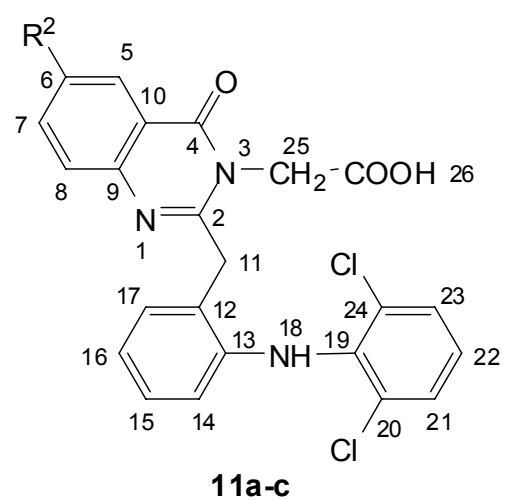

Fig. 6. Numbering of Quinazolin-4(3H)-ones 11a-c

[2-\{2-[(2,6-Dichlorophenyl)amino]benzyl\}-4-oxoquinazolin-3(4H)-yl]acetic acid (11a)

Yield: 53\%, mp: $202-206^{\circ} \mathrm{C}$. IR (KBr), v, cm ${ }^{-1}: 741(\mathrm{C}-\mathrm{Cl}), 1315(\mathrm{C}-\mathrm{N}), 1608(\mathrm{C}=\mathrm{N}$ quinazolinone), $1686\left(\mathrm{C}=\mathrm{O}\right.$ quinazolinone), $1715(\mathrm{C}=\mathrm{O}), 2780(\mathrm{OH}), 2924,2851\left(\mathrm{CH}_{2}\right)$, $3447(\mathrm{NH})$. ${ }^{1} \mathrm{H}$ NMR (400 MHz, DMSO-d $d_{6}$, TMS): $\delta 3.52$ (s, 2H, H-11), 4.15 (s, 2H, H-25), $6.39(\mathrm{~d}, J=7.96 \mathrm{~Hz}, 1 \mathrm{H}, \mathrm{H}-14), 6.88(\mathrm{t}, J=7.44 \mathrm{~Hz}, 1 \mathrm{H}, \mathrm{H}-16), 7.04-7.09(\mathrm{~m}, 2 \mathrm{H}, \mathrm{H}-$ 15,22), $7.21(\mathrm{~d}, J=7.58 \mathrm{~Hz}, 1 \mathrm{H}, \mathrm{H}-17), 7.42(\mathrm{~d}, J=8.08 \mathrm{~Hz}, 2 \mathrm{H}, \mathrm{H}-21,23), 7.48(\mathrm{t}, J=$ $7.72 \mathrm{~Hz}, 1 \mathrm{H}, \mathrm{H}-6), 7.62(\mathrm{~d}, J=8.2 \mathrm{~Hz}, 1 \mathrm{H}, \mathrm{H}-8), 7.75(\mathrm{t}, J=7.88 \mathrm{~Hz}, 1 \mathrm{H}, \mathrm{H}-7), 8.10$ (d, J = $7.8 \mathrm{~Hz}, 1 \mathrm{H}, \mathrm{H}-5), 9.10$ (bs, 1H, H-18), 12.34 (bs, 1H, H-26). ${ }^{13} \mathrm{C}$ NMR $(100 \mathrm{MHz}$, DMSO$\left.d_{6}, \mathrm{TMS}\right): \delta 32.62$ (C-11), 42.93 (C-25), 116.31 (C-16), 120.44 (C-14), 120.91 (C-10), 122.52 (C-8), 124.29 (C-22), 126.51 (C-15), 127.18 (C-12), 127.37 (C-21,23), 127.82 (C6), 128.71 (C-5), 129.32 (C-20,24), 131.14 (C-17), 135.74 (C-7), 137.28 (C-19), 141.71 (C13), 147.13 (C-9), 160.72 (C-4), 164.63 (C-2), 173.52 (C-26). Anal. Calcd. for $\mathrm{C}_{23} \mathrm{H}_{17} \mathrm{Cl}_{2} \mathrm{~N}_{3} \mathrm{O}_{3}: \mathrm{C}, 60.81 ; \mathrm{H}, 3.77 ; \mathrm{N}, 9.25$. Found: $\mathrm{C}, 60.75 ; \mathrm{H}, 3.70 ; \mathrm{N}, 9.28$.

[6-Bromo-2-\{2-[(2,6-dichlorophenyl)amino]benzy/\}-4-oxoquinazolin-3(4H)-yl]acetic acid (11b)

Yield: 56\%, mp: 223-227 ${ }^{\circ} \mathrm{C}$. IR (KBr), v, cm ${ }^{-1}: 567$ (C-Br), 744 (C-Cl), 1317 (C-N), 1610 $(\mathrm{C}=\mathrm{N}$ quinazolinone), $1684(\mathrm{C}=\mathrm{O}$ quinazolinone), $1718(\mathrm{C}=\mathrm{O}), 2785(\mathrm{OH}), 2925,2849$ $\left(\mathrm{CH}_{2}\right), 3445(\mathrm{NH}) .{ }^{1} \mathrm{H}$ NMR (400 MHz, DMSO-d 6 , TMS): $\delta 3.53(\mathrm{~s}, 2 \mathrm{H}, \mathrm{H}-11), 4.14(\mathrm{~s}, 2 \mathrm{H}$, $\mathrm{H}-25), 6.40(\mathrm{~d}, J=8 \mathrm{~Hz}, 1 \mathrm{H}, \mathrm{H}-14), 6.88(\mathrm{t}, J=7.4 \mathrm{~Hz}, 1 \mathrm{H}, \mathrm{H}-16), 7.03-7.08(\mathrm{~m}, 2 \mathrm{H}, \mathrm{H}-$ 15,22), 7.22 (d, $J=7.5 \mathrm{~Hz}, 1 \mathrm{H}, \mathrm{H}-17$ ), 7.41 (d, $J=8.12 \mathrm{~Hz}, 2 \mathrm{H}, \mathrm{H}-21,23), 7.63$ (d, $J=8.36$ 
$\mathrm{Hz}, 1 \mathrm{H}, \mathrm{H}-8), 7.95$ (d, J = 8.36 Hz, 1H, H-7), 8.03 (s, 1H, H-5), 9.09 (bs, 1H, H-18), 12.35 (bs, $1 \mathrm{H}, \mathrm{H}-26) .{ }^{13} \mathrm{C}$ NMR (100 MHz, DMSO-d 6 , TMS): $\delta 32.73$ (C-11), 43.32 (C-25), 116.28 (C-16), 120.57 (C-14), 121.65 (C-6), 122.94 (C-10), 124.19 (C-22), 124.51 (C-8), 126.42 (C-15), 127.15 (C-12), 127.38 (C-21,23), 129.31 (C-20,24), 131.22 (C-17), 132.14 (C-5), 137.37 (C-19), 138.33 (C-7), 141.81 (C-13), 145.83 (C-9), 160.93 (C-4), 164.55 (C-2), 173.47 (C-26). Anal. Calcd. for $\mathrm{C}_{23} \mathrm{H}_{16} \mathrm{BrCl}_{2} \mathrm{~N}_{3} \mathrm{O}_{3}$ : C, 51.81; $\mathrm{H}, 3.02 ; \mathrm{N}, 7.88$. Found: C, $51.85 ; \mathrm{H}, 3.05 ; \mathrm{N}, 7.84$.

[2-\{2-[(2,6-Dichlorophenyl)amino]benzyl\}-6-iodo-4-oxoquinazolin-3(4H)-yl]acetic acid (11c) Yield: $60 \%, \mathrm{mp}: 211-214^{\circ} \mathrm{C} . \mathrm{IR}(\mathrm{KBr}), \mathrm{v}, \mathrm{cm}^{-1}: 618(\mathrm{C}-\mathrm{I}), 746(\mathrm{C}-\mathrm{Cl}), 1318$ (C-N), 1612 ( $\mathrm{C}=\mathrm{N}$ quinazolinone), $1682(\mathrm{C}=\mathrm{O}$ quinazolinone), $1713(\mathrm{C}=\mathrm{O}), 2778(\mathrm{OH}), 2922,2846$ $\left(\mathrm{CH}_{2}\right), 3443(\mathrm{NH}) .{ }^{1} \mathrm{H}$ NMR (400 MHz, DMSO-d, TMS): $\delta 3.53$ (s, 2H, H-11), 4.16 (s, 2H, $\mathrm{H}-25), 6.41$ (d, J = 7.92 Hz, 1H, H-14), 6.89 (t, J = 7.36 Hz, 1H, H-16), 7.04-7.09 (m, 2H, $\mathrm{H}-15,22), 7.22$ (d, J = 7.5 Hz, 1H, H-17), 7.26 (d, J = 8.32 Hz, 1H, H-8), 7.42 (d, J = 8.04 $\mathrm{Hz}, 2 \mathrm{H}, \mathrm{H}-21,23), 7.94$ (d, J = 8.32 Hz, 1H, H-7), 8.27 (s, 1H, H-5), 9.09 (bs, 1H, H-18), 12.35 (bs, $1 \mathrm{H}, \mathrm{H}-26) .{ }^{13} \mathrm{C}$ NMR (100 MHz, DMSO-d 6 , TMS): $\delta 33.12$ (C-11), 43.26 (C-25), 93.32 (C-6), 116.26 (C-16), 120.51 (C-14), 122.52 (C-10), 123.92 (C-8), 124.13 (C-22), 126.58 (C-15), 126.98 (C-12), 127.26 (C-21,23), 129.32 (C-20,24), 131.24 (C-17), 136.14 (C-5), 137.28 (C-19), 141.67 (C-13), 142.45 (C-7), 145.74 (C-9), 161.16 (C-4), 164.62 (C2), 173.44 (C-26). Anal. Calcd. for $\mathrm{C}_{23} \mathrm{H}_{16} \mathrm{Cl}_{2} \mathrm{IN}_{3} \mathrm{O}_{3}$ : $\mathrm{C}, 47.61 ; \mathrm{H}, 2.78 ; \mathrm{N}, 7.24$. Found: $\mathrm{C}$, $47.57 ; \mathrm{H}, 2.75 ; \mathrm{N}, 7.25$.

\section{General procedure for 1,3,4-oxadiazolyl-quinazolin-4(3H)-ones (12a-f)}

A mixture of $11 \mathbf{a}-\mathbf{c}(2.5 \mathrm{mmol})$, benzohydrazides $3 \mathbf{a}, \mathbf{b}(2.5 \mathrm{mmol})$ and $7 \mathrm{ml}$ phosphorus trichloride in $10 \mathrm{ml}$ dry benzene was refluxed under anhydrous condition for $10-12 \mathrm{~h}$. After completion of reaction, benzene was distilled off under reduced pressure and the residue poured on to crushed ice and neutralized with sodium bicarbonate $(5 \% \mathrm{w} / \mathrm{v})$. The solid thus obtained was filtered, washed with cold water and recrystallized from ethanol.

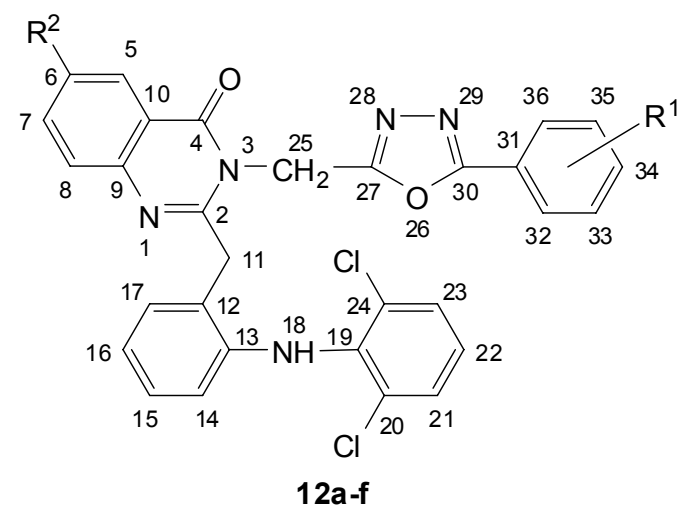

Fig. 7. Numbering of 1,3,4-Oxadiazolyl-quinazolin-4(3H)-ones $\mathbf{1 2 a - f}$

3-\{[5-(2-Chlorophenyl)-1,3,4-oxadiazol-2-yl]methyl\}-2-\{2-[(2,6-dichlorophenyl)amino]benzyl\}quinazolin-4(3H)-one (12a)

Yield: $72 \%, \mathrm{mp}: 181-184^{\circ} \mathrm{C} . \mathrm{IR}(\mathrm{KBr}), \mathrm{v}, \mathrm{cm}^{-1}: 744(\mathrm{C}-\mathrm{Cl}), 1313$ (C-N), 1259, 1035 (C-O-C oxadiazole), $1611 \quad(\mathrm{C}=\mathrm{N}$ quinazolinone $), \quad 1653 \quad(\mathrm{C}=\mathrm{N}$ oxadiazole $), \quad 1678 \quad(\mathrm{C}=\mathrm{O}$ 
quinazolinone), 2923, $2855\left(\mathrm{CH}_{2}\right), 3448(\mathrm{NH}) .{ }^{1} \mathrm{H}$ NMR (400 MHz, DMSO-d 6 , TMS): $\delta 3.51$ (s, 2H, H-11), 4.49 (s, 2H, H-25), 6.40 (d, J = 7.96 Hz, 1H, H-14), 6.89 (t, J = 7.44 Hz, 1H, $\mathrm{H}-16), 7.05-7.10(\mathrm{~m}, 2 \mathrm{H}, \mathrm{H}-15,22), 7.22(\mathrm{~d}, J=7.58 \mathrm{~Hz}, 1 \mathrm{H}, \mathrm{H}-17), 7.42(\mathrm{~d}, J=8.12 \mathrm{~Hz}$, $2 \mathrm{H}, \mathrm{H}-21,23), 7.47(\mathrm{t}, J=7.56 \mathrm{~Hz}, 1 \mathrm{H}, \mathrm{H}-34), 7.51(\mathrm{t}, J=7.64 \mathrm{~Hz}, 1 \mathrm{H}, \mathrm{H}-6), 7.56$ (t, J = 7.4 $\mathrm{Hz}, 1 \mathrm{H}, \mathrm{H}-35), 7.61(\mathrm{~d}, \mathrm{~J}=8.12 \mathrm{~Hz}, 1 \mathrm{H}, \mathrm{H}-8), 7.67(\mathrm{~d}, J=7.84 \mathrm{~Hz}, 1 \mathrm{H}, \mathrm{H}-33), 7.71(\mathrm{~d}, J=$ $7.48 \mathrm{~Hz}, 1 \mathrm{H}, \mathrm{H}-36), 7.75$ (t, J = 7.8 Hz, 1H, H-7), 8.09 (d, J = 7.72 Hz, 1H, H-5), 9.10 (bs, $1 \mathrm{H}, \mathrm{H}-18) .{ }^{13} \mathrm{C}$ NMR (100 MHz, DMSO-d 6 , TMS): $\delta 33.02$ (C-11), 42.42 (C-25), 116.32 (C16), 120.45 (C-14), 120.93 (C-10), 122.57 (C-8), 124.31 (C-22), 126.54 (C-15), 127.22 (C12), 127.39 (C-21,23), 127.61 (C-35), 127.83 (C-6), 128.24 (C-36), 128.73 (C-5), 128.82 (C-33), 129.37 (C-20,24), 129.75 (C-34), 131.18 (C-17), 131.79 (C-32), 135.47 (C-31), 135.81 (C-7), 137.34 (C-19), 141.75 (C-13), 147.18 (C-9), 156.28 (C-27), 160.74 (C-4), 163.46 (C-2), 164.65 (C-30). Anal. Calcd. for $\mathrm{C}_{30} \mathrm{H}_{20} \mathrm{Cl}_{3} \mathrm{~N}_{5} \mathrm{O}_{2}$ : C, 61.19; $\mathrm{H}, 3.42 ; \mathrm{N}, 11.89$. Found: C, 61.12; H, 3.47; N, 11.85.

\section{6-Bromo-3-\{[5-(2-chlorophenyl)-1,3,4-oxadiazol-2-yl]methyl\}-2-\{2-[(2,6-dichlorophenyl)- amino]benzyl\}quinazolin-4(3H)-one (12b)}

Yield: $68 \%$, mp: $175-178^{\circ} \mathrm{C}$. IR (KBr), v, cm ${ }^{-1}: 568$ (C-Br), 742 (C-Cl), 1311 (C-N), 1263, 1045 (C-O-C oxadiazole), 1612 (C=N quinazolinone), 1653 ( $\mathrm{C}=\mathrm{N}$ oxadiazole), 1675 ( $\mathrm{C}=\mathrm{O}$ quinazolinone), 2922, $2850\left(\mathrm{CH}_{2}\right), 3443(\mathrm{NH}) .{ }^{1} \mathrm{H}$ NMR (400 MHz, DMSO-d $\left.d_{6}, \mathrm{TMS}\right): \delta 3.53$ (s, 2H, H-11), $4.48(\mathrm{~s}, 2 \mathrm{H}, \mathrm{H}-25), 6.40(\mathrm{~d}, J=8 \mathrm{~Hz}, 1 \mathrm{H}, \mathrm{H}-14), 6.88(\mathrm{t}, J=7.48 \mathrm{~Hz}, 1 \mathrm{H}, \mathrm{H}-$ 16), 7.03-7.08 (m, 2H, H-15,22), 7.22 (d, J = 7.58 Hz, $1 \mathrm{H}, \mathrm{H}-17), 7.41(\mathrm{~d}, J=8.08 \mathrm{~Hz}, 2 \mathrm{H}$, $\mathrm{H}-21,23), 7.49$ (t, J = 7.52 Hz, 1H, H-34), 7.56 (t, $J=7.36 \mathrm{~Hz}, 1 \mathrm{H}, \mathrm{H}-35), 7.62$ (d, $J=8.28$ $\mathrm{Hz}, 1 \mathrm{H}, \mathrm{H}-8), 7.67$ (d, J = 7.8 Hz, 1H, H-33), 7.72 (d, J = 7.48 Hz, 1H, H-36), 8.06 (d, J = $8.28 \mathrm{~Hz}, 1 \mathrm{H}, \mathrm{H}-7), 8.11(\mathrm{~s}, 1 \mathrm{H}, \mathrm{H}-5), 9.10$ (bs, $1 \mathrm{H}, \mathrm{H}-18) .{ }^{13} \mathrm{C}$ NMR (100 MHz, DMSO-d 6 , TMS): $\delta 33.14$ (C-11), 42.47 (C-25), 116.32 (C-16), 120.63 (C-14), 121.68 (C-6), 122.96 (C-10), 124.22 (C-22), 124.54 (C-8), 126.46 (C-15), 127.17 (C-12), 127.42 (C-21,23), 127.67 (C-35), 128.26 (C-36), 128.72 (C-33), 129.35 (C-20,24), 129.69 (C-34), 131.24 (C17), 131.84 (C-32), 132.16 (C-5), 135.52 (C-31), 137.43 (C-19), 138.37 (C-7), 141.84 (C13), 145.85 (C-9), 156.25 (C-27), 160.97 (C-4), 163.41 (C-2), 164.56 (C-30). Anal. Calcd. for $\mathrm{C}_{30} \mathrm{H}_{19} \mathrm{BrCl}_{3} \mathrm{~N}_{5} \mathrm{O}_{2}: \mathrm{C}, 53.96 ; \mathrm{H}, 2.87 ; \mathrm{N}, 10.49$. Found: $\mathrm{C}, 53.88 ; \mathrm{H}, 2.85 ; \mathrm{N}, 10.53$.

3-\{[5-(2-Clorophenyl)-1,3,4-oxadiazol-2-yl]methyl\}-2-\{2-[(2, 6-dichlorophenyl)amino]benzyl\}6-iodoquinazolin-4(3H)-one (12c)

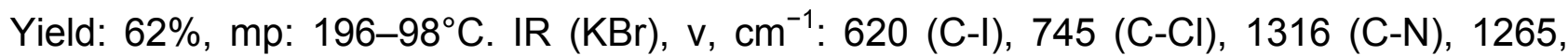
1036 (C-O-C oxadiazole), 1609 ( $\mathrm{C}=\mathrm{N}$ quinazolinone), 1650 ( $\mathrm{C}=\mathrm{N}$ oxadiazole), 1678 ( $\mathrm{C}=\mathrm{O}$ quinazolinone), 2924, $2850\left(\mathrm{CH}_{2}\right), 3445(\mathrm{NH}) .{ }^{1} \mathrm{H}$ NMR (400 MHz, DMSO-d $\left.d_{6}, \mathrm{TMS}\right): \delta 3.53$ (s, 2H, H-11), $4.48(\mathrm{~s}, 2 \mathrm{H}, \mathrm{H}-25), 6.41(\mathrm{~d}, J=8 \mathrm{~Hz}, 1 \mathrm{H}, \mathrm{H}-14), 6.89(\mathrm{t}, J=7.48 \mathrm{~Hz}, 1 \mathrm{H}, \mathrm{H}-$ 16), 7.04-7.09 (m, 2H, H-15,22), $7.22(\mathrm{~d}, J=7.62 \mathrm{~Hz}, 1 \mathrm{H}, \mathrm{H}-17), 7.26(\mathrm{~d}, J=8.36 \mathrm{~Hz}, 1 \mathrm{H}$, $\mathrm{H}-8), 7.42(\mathrm{~d}, J=8.12 \mathrm{~Hz}, 2 \mathrm{H}, \mathrm{H}-21,23), 7.50(\mathrm{t}, J=7.52 \mathrm{~Hz}, 1 \mathrm{H}, \mathrm{H}-34), 7.56$ (t, $J=7.36$ $\mathrm{Hz}, 1 \mathrm{H}, \mathrm{H}-35), 7.65$ (d, J = 7.8 Hz, 1H, H-33), 7.71 (d, J = 7.44 Hz, 1H, H-36), $7.94(\mathrm{~d}, J=$ $8.36 \mathrm{~Hz}, 1 \mathrm{H}, \mathrm{H}-7), 8.27$ (s, $1 \mathrm{H}, \mathrm{H}-5), 9.09$ (bs, $1 \mathrm{H}, \mathrm{H}-18) .{ }^{13} \mathrm{C}$ NMR (100 MHz, DMSO-d 6 , TMS): $\delta 33.51$ (C-11), 42.47 (C-25), 93.33 (C-6), 116.27 (C-16), 120.53 (C-14), 122.55 (C10), 123.91 (C-8), 124.16 (C-22), 126.62 (C-15), 127.11 (C-12), 127.28 (C-21,23), 127.64 (C-35), 128.26 (C-36), 128.73 (C-33), 129.37 (C-20,24), 129.75 (C-34), 131.28 (C-17), 131.84 (C-32), 136.15 (C-5), 135.52 (C-31), 137.31 (C-19), 141.70 (C-13), 142.48 (C-7), 145.77 (C-9), 156.23 (C-27), 161.18 (C-4), 163.44 (C-2), 164.67 (C-30). Anal. Calcd. for $\mathrm{C}_{30} \mathrm{H}_{19} \mathrm{Cl}_{3} \mathrm{IN}_{5} \mathrm{O}_{2}: \mathrm{C}, 50.41 ; \mathrm{H}, 2.68 ; \mathrm{N}, 9.80$. Found: $\mathrm{C}, 50.45 ; \mathrm{H}, 2.71 ; \mathrm{N}, 9.73$. 
3-\{[5-(4Chlorophenyl)-1,3,4-oxadiazol-2-yl]methyl\}-2-\{2-[(2,6-dichlorophenyl)amino]benzyl\}quinazolin-4(3H)-one (12d)

Yield: 71\%, mp: 227-30 C. IR (KBr), v, cm ${ }^{-1}$ : 748 (C-Cl), 1316 (C-N), 1254, 1038 (C-O-C oxadiazole), $1608 \quad(\mathrm{C}=\mathrm{N}$ quinazolinone), $1655 \quad(\mathrm{C}=\mathrm{N}$ oxadiazole), $1683 \quad(\mathrm{C}=\mathrm{O}$ quinazolinone), 2922, $2851\left(\mathrm{CH}_{2}\right), 3443(\mathrm{NH}) .{ }^{1} \mathrm{H}$ NMR (400 MHz, DMSO-d 6 , TMS): $\delta 3.52$ (s, 2H, H-11), 4.47 (s, 2H, H-25), 6.39 (d, J = 8.04 Hz, 1H, H-14), 6.88 (t, J = 7.52 Hz, 1H, $\mathrm{H}-16), 7.04-7.09(\mathrm{~m}, 2 \mathrm{H}, \mathrm{H}-15,22), 7.21(\mathrm{~d}, J=7.62 \mathrm{~Hz}, 1 \mathrm{H}, \mathrm{H}-17), 7.42(\mathrm{~d}, J=8.16 \mathrm{~Hz}$, $2 \mathrm{H}, \mathrm{H}-21,23), 7.50$ (t, J = 7.68 Hz, 1H, H-6), 7.62 (d, J = 8.16 Hz, 1H, H-8), 7.65 (d, J = 8.2 $\mathrm{Hz}, 2 \mathrm{H}, \mathrm{H}-33,35), 7.70$ (d, J = 8.2 Hz, 2H, H-32,36), 7.74 (t, J = 7.84 Hz, 1H, H-7), 8.08 (d, $J=7.76 \mathrm{~Hz}, 1 \mathrm{H}, \mathrm{H}-5), 9.12$ (bs, $1 \mathrm{H}, \mathrm{H}-18) .{ }^{13} \mathrm{C}$ NMR (100 MHz, DMSO-d 6 , TMS): $\delta 33.05$ (C-11), 42.23 (C-25), 116.35 (C-16), 120.48 (C-14), 120.96 (C-10), 122.55 (C-8), 123.34 (C-31), 124.34 (C-22), 126.53 (C-15), 127.26 (C-12), 127.34 (C-21,23), 127.77 (C-6), 128.74 (C-5), 129.35 (C-20,24), 131.21 (C-17), 131.83 (C-32,36), 133.26 (C-33,35), 135.38 (C-34), 135.78 (C-7), 137.36 (C-19), 141.76 (C-13), 147.18 (C-9), 156.27 (C-27), 160.78 (C-4), 163.38 (C-2), 164.67 (C-30). Anal. Calcd. for $\mathrm{C}_{30} \mathrm{H}_{20} \mathrm{Cl}_{3} \mathrm{~N}_{5} \mathrm{O}_{2}$ : C, 61.19; $\mathrm{H}$, 3.42; N, 11.89. Found: C, 61.22; H, 3.38; N, 11.84.

6-Bromo-3-\{[5-(4chlorophenyl)-1,3,4-oxadiazol-2-yl]methyl\}-2-\{2-[(2,6-dichlorophenyl)amino]benzyl\}quinazolin-4(3H)-one (12e)

Yield: $67 \%, \mathrm{mp}: 266-69^{\circ} \mathrm{C}$. IR (KBr), v, cm ${ }^{-1}: 571$ (C-Br), 750 (C-Cl), $1313(\mathrm{C}-\mathrm{N}), 1263$, 1041 (C-O-C oxadiazole), 1607 ( $\mathrm{C}=\mathrm{N}$ quinazolinone), 1656 ( $\mathrm{C}=\mathrm{N}$ oxadiazole), 1678 ( $\mathrm{C}=\mathrm{O}$ quinazolinone), 2925, $2853\left(\mathrm{CH}_{2}\right), 3448(\mathrm{NH}) .{ }^{1} \mathrm{H}$ NMR (400 MHz, DMSO-d 6 , TMS): $\delta 3.53$ (s, 2H, H-11), 4.49 (s, 2H, H-25), 6.40 (d, J = 7.88 Hz, 1H, H-14), 6.89 (t, J = 7.32 Hz, 1H, $\mathrm{H}-16), 7.02-7.08(\mathrm{~m}, 2 \mathrm{H}, \mathrm{H}-15,22), 7.20(\mathrm{~d}, J=7.46 \mathrm{~Hz}, 1 \mathrm{H}, \mathrm{H}-17), 7.42(\mathrm{~d}, J=8.04 \mathrm{~Hz}$, $2 \mathrm{H}, \mathrm{H}-21,23), 7.60(\mathrm{~d}, J=8.32 \mathrm{~Hz}, 1 \mathrm{H}, \mathrm{H}-8), 7.65$ (d, J = 8.24 Hz, 2H, H-33,35), 7.72 (d, J $=8.24 \mathrm{~Hz}, 2 \mathrm{H}, \mathrm{H}-32,36), 8.05(\mathrm{~d}, \mathrm{~J}=8.32 \mathrm{~Hz}, 1 \mathrm{H}, \mathrm{H}-7), 8.12(\mathrm{~s}, 1 \mathrm{H}, \mathrm{H}-5), 9.08(\mathrm{bs}, 1 \mathrm{H}, \mathrm{H}-$ 18). ${ }^{13} \mathrm{C}$ NMR (100 MHz, DMSO- $d_{6}$, TMS): $\delta 33.15$ (C-11), 42.51 (C-25), 116.25 (C-16), 120.54 (C-14), 121.63 (C-6), 122.87 (C-10), 123.30 (C-31), 124.15 (C-22), 124.46 (C-8), 126.38 (C-15), 127.17 (C-12), 127.42 (C-21,23), 129.28 (C-20,24), 131.25 (C-17), 131.78 (C-32,36), 132.12 (C-5), 133.23 (C-33,35), 135.46 (C-34), 137.42 (C-19), 138.32 (C-7), 141.83 (C-13), 145.85 (C-9), 156.31 (C-27), 160.94 (C-4), 163.37 (C-2), 164.57 (C-30). Anal. Calcd. for $\mathrm{C}_{30} \mathrm{H}_{19} \mathrm{BrCl}_{3} \mathrm{~N}_{5} \mathrm{O}_{2}$ : C, 53.96; $\mathrm{H}, 2.87 ; \mathrm{N}, 10.49$. Found: $\mathrm{C}, 53.91 ; \mathrm{H}, 2.82 ; \mathrm{N}$, 10.48.

3-\{[5-(4Clorophenyl)-1,3,4-oxadiazol-2-yl]methyl\}-2-\{2-[(2, 6-dichlorophenyl)amino]benzyl\}6-iodoquinazolin-4(3H)-one (12f)

Yield: $75 \%, \mathrm{mp}: 243-46^{\circ} \mathrm{C} . \mathrm{IR}(\mathrm{KBr}), \mathrm{v}, \mathrm{cm}^{-1}: 618$ (C-I), 749 (C-Cl), 1315 (C-N), 1260, 1049 (C-O-C oxadiazole), 1611 ( $\mathrm{C}=\mathrm{N}$ quinazolinone), 1658 ( $\mathrm{C}=\mathrm{N}$ oxadiazole), 1683 ( $\mathrm{C}=\mathrm{O}$ quinazolinone), 2921, $2849\left(\mathrm{CH}_{2}\right), 3445(\mathrm{NH}) .{ }^{1} \mathrm{H}$ NMR (400 MHz, DMSO-d 6 , TMS): $\delta 3.51$ (s, 2H, H-11), $4.48(\mathrm{~s}, 2 \mathrm{H}, \mathrm{H}-25), 6.42(\mathrm{~d}, J=7.96 \mathrm{~Hz}, 1 \mathrm{H}, \mathrm{H}-14), 6.90$ (t, J = 7.4 Hz, $1 \mathrm{H}$, $\mathrm{H}-16), 7.03-7.08(\mathrm{~m}, 2 \mathrm{H}, \mathrm{H}-15,22), 7.21(\mathrm{dd}, J=7.5 \mathrm{~Hz}, 1.28 \mathrm{~Hz}, 1 \mathrm{H}, \mathrm{H}-17), 7.24(\mathrm{~d}, J=$ $8.4 \mathrm{~Hz}, 1 \mathrm{H}, \mathrm{H}-8), 7.41(\mathrm{~d}, J=8.12 \mathrm{~Hz}, 2 \mathrm{H}, \mathrm{H}-21,23), 7.62$ (d, J = 8.2 Hz, 2H, H-33,35), 7.68 (d, J = 8.2 Hz, 2H, H-32,36), 7.93 (d, J=8.4 Hz, 1H, H-7), 8.28 (s, 1H, H-5), 9.12 (bs, $1 \mathrm{H}, \mathrm{H}-18) .{ }^{13} \mathrm{C}$ NMR (100 MHz, DMSO-d $\left.d_{6}, \mathrm{TMS}\right): \delta 33.47$ (C-11), 42.45 (C-25), 93.38 (C6), 116.31 (C-16), 120.56 (C-14), 122.54 (C-10), 123.33 (C-31), 123.97 (C-8), 124.17 (C22), 126.60 (C-15), 127.02 (C-12), 127.25 (C-21,23), 129.36 (C-20,24), 131.27 (C-17), 131.77 (C-32,36), 133.29 (C-33,35), 135.45 (C-34), 136.18 (C-5), 137.33 (C-19), 141.71 
(C-13), 142.48 (C-7), 145.76 (C-9), 156.31 (C-27), 161.14 (C-4), 163.49 (C-2), 164.63 (C30). Anal. Calcd. for $\mathrm{C}_{30} \mathrm{H}_{19} \mathrm{Cl}_{3} \mathrm{IN}_{5} \mathrm{O}_{2}$ : C, 50.41; $\mathrm{H}, 2.68 ; \mathrm{N}, 9.80$. Found: $\mathrm{C}, 50.35 ; \mathrm{H}, 2.76$; N, 9.77 .

\section{Antimicrobial activity}

The MICs of synthesized compounds were carried out by broth microdilution method as described by Rattan [35]. Antibacterial activity was screened against two gram positive bacteria ( $S$. aureus MTCC 96, $S$. pyogenes MTCC 442) and two gram negative bacteria (E. coli MTCC 443, P. aeruginosa MTCC 1688). Ampicillin was used as a standard antibacterial agent. Antifungal activity was screened against three fungal species $C$. albicans MTCC 227, A. niger MTCC 282 and A. clavatus MTCC 1323. Griseofulvin was used as a standard antifungal agent.

All MTCC cultures were collected from Institute of Microbial Technology, Chandigarh and tested against above mentioned known drugs. Mueller hinton broth was used as nutrient medium to grow and dilute the drug suspension for the test. Inoculum size for test strain was adjust to $10^{8} \mathrm{CFU}$ (Colony Forming Unit) per milliliter by comparing the turbidity. DMSO was used as diluents to get desired concentration of drugs to test upon standard bacterial strains. Serial dilutions were prepared in primary and secondary screening. The control tube containing no antibiotic was immediately sub cultured (before inoculation) by spreading a loopful evenly over a quarter of plate of medium suitable for the growth of the test organism and put for incubation at $37^{\circ} \mathrm{C}$ overnight. The tubes were then incubated overnight. The MIC of the control organism was read to check the accuracy of the drug concentrations. The lowest concentration inhibiting growth of the organism was recorded as the MIC. All the tubes not showing visible growth (in the same manner as control tube described above) was sub cultured and incubated overnight at $37^{\circ} \mathrm{C}$. The amount of growth from the control tube before incubation (which represents the original inoculum) was compared. Subcultures might show: similar number of colonies indicating bacteriostatic; a reduced number of colonies indicating a partial or slow bactericidal activity and no growth if the whole inoculum has been killed. The test must include a second set of the same dilutions inoculated with an organism of known sensitivity. Each synthesized drug was diluted obtaining $2000 \mu \mathrm{g} / \mathrm{ml}$ concentration, as a stock solution. In primary screening $500 \mu \mathrm{g} / \mathrm{ml}, 250 \mu \mathrm{g} / \mathrm{ml}$ and $125 \mu \mathrm{g} / \mathrm{ml}$ concentrations of the synthesized drugs were taken. The active synthesized drugs found in this primary screening were further tested in a second set of dilution against all microorganisms. The drugs found active in primary screening were similarly diluted to obtain $100 \mu \mathrm{g} / \mathrm{ml}, 50 \mu \mathrm{g} / \mathrm{ml}, 25 \mu \mathrm{g} / \mathrm{ml}, 12.5$ $\mu \mathrm{g} / \mathrm{ml}, 6.250 \mu \mathrm{g} / \mathrm{ml}, 3.125 \mu \mathrm{g} / \mathrm{ml}$ and $1.5625 \mu \mathrm{g} / \mathrm{ml}$ concentrations. The highest dilution showing at least $99 \%$ inhibition is taken as MIC.

\section{Conclusions}

Aminosubstituted 1,3,4-oxadiazoles $\mathbf{4 a , b}$ and $\mathbf{5 a , b}$ exhibited very good antimicrobial activity. But when they were condensed with benzoxazinone formed oxadiazolylquinazolinone, showed increasing activity. Antimicrobial results were found uneven but most of the bromo and iodo derivatives of quinazolinone possessed very good antimicrobial activity. Furthermore $\mathrm{CH}_{2}$ link between $3^{\text {rd }}$ position of quinazolinone and $2^{\text {nd }}$ position of oxadiazole were found most active than other two series. So, it seems from the 
antimicrobial results that halogen atom and $\mathrm{CH}_{2}$ link played vital role in increasing antimicrobial activity.

\section{Acknowledgement}

The authors are thankful to Veer Narmad South Gujarat University for providing necessary facilities, CDRI Lucknow and SAIF Chandigarh for spectral analysis of the compounds. Thank to Dhanji Rajani for antimicrobial activity screening.

\section{Authors' Statement}

\section{Competing Interests}

The authors declare no conflict of interest.

\section{References}

[1] Zheng X, Li Z, Wang Y, Chen W, Huang Q, Liu C, Song G.

Syntheses and insecticidal activities of novel 2,5-disubstituted 1,3,4-oxadiazoles.

J Fluorine Chem. 2003; 123: 163-169.

doi:10.1016/S0022-1139(03)00168-4

[2] Chavan VP, Sonawane SA, Shingare MS, Karale BK.

Synthesis, characterization, and biological activities of some 3,5,6-trichloropyridine derivatives.

Chem Heterocyclic Compounds. 2006; 42: 625-630.

doi:10.1007/s10593-006-0137-8

[3] Hollaa BS, Gonsalves R, Shenoy S.

Synthesis and antibacterial studies of a new series of 1,2-bis(1,3,4-oxadiazol-2-yl)ethanes and 1,2bis(4-amino-1,2,4-triazol-3-yl)ethanes.

Eur J Med Chem. 2000; 35: 267-271. doi:10.1016/S0223-5234(00)00154-9

[4] Liu F, Luo X, Song B, Bhadury PS, Yang S, Jin L, Xue W, Hu D.

Synthesis and antifungal activity of novel sulfoxide derivatives containing trimethoxyphenyl substituted 1,3,4-thiadiazole and 1,3,4-oxadiazole moiety.

Bioorg Med Chem. 2008; 16: 3632-3640.

doi:10.1016/j.bmc.2008.02.006

[5] Narayana B, Raj KKV, Ashalatha BV, Kumari NS.

Synthesis of some new 2-(6-methoxy-2-naphthyl)-5-aryl-1,3,4-oxadiazoles as possible non-steroidal anti-inflammatory and analgesic agents.

Arch Pharm. 2005; 338: 373-377.

doi:10.1002/ardp.200500974

[6] Koksal M, Bilge SS, Bozkurt A, Sahin ZS, Isik S, Erol DD.

Synthesis, characterization and anti-inflammatory activity of new 5-(3,4-dichlorophenyl)-2-(aroyl methyl)thio-1,3,4-oxadiaxoles.

Arzneimittel-Forschung. 2008; 58: 510-514.

[7] Zareef M, Iqbal R, Dominguez NG, Rodrigues J, Zaidi JH, Arfan M, Supuran CT.

Synthesis and antimalarial activity of novel chiral and achiral benzenesulfonamides bearing 1,3,4oxadiazole moieties.

J Enz Inhib Med Chem. 2007; 22: 301-308.

doi:10.1080/14756360601114569

[8] Farghaly AR, El-Kashef $\mathrm{H}$.

Synthesis of some new azoles with antiviral potential ${ }^{1}$.

Arkivoc. 2006; 11: 76-90. 
[9] El-Essawy FA, Khattab AF, Abdel-Rahman AAH.

Synthesis of 1,2,4-triazol-3-ylmethyl-, 1,3,4-oxa-, and -thiadiazol-2-ylmethyl-1H-[1,2,3]-triazolo[4,5d]pyrimidinediones.

Monatsh Chem. 2007; 138: 777-785.

doi:10.1007/s00706-007-0649-7

[10] Amr AEE, Mohamed SF, Abdel-Hafez NA, Abdalla MM.

Antianexiety activity of pyridine derivatives synthesized from 2-chloro-6-hydrazino-isonicotinic acid hydrazide.

Monatsh Chem. 2008; 139: 1491-1498.

doi:10.1007/s00706-008-0949-6

[11] Kumar A, D'Souza SS, Gaonkar SL, Rai KML, Salimath BP.

Growth inhibition and induction of apoptosis in MCF-7 breast cancer cells by a new series of substituted-1,3,4-oxadiazole derivatives.

Invest New Drugs. 2008; 26: 425-435.

doi:10.1007/s10637-008-9116-5

[12] Zareef M, Iqbal R, Al-Masoudi NA, Zaidi JH, Arfan M, Shahzad SA.

Synthesis, anti-HIV, and antifungal activity of new benzensulfonamides bearing the 2,5-disubstituted1,3,4-oxadiazole moiety.

Phosphorus Sulfur and Silicon. 2007; 182: 281-298.

doi:10.1080/10426500600919074

[13] Macaev F, Rusu G, Pogrebnoi S, Gudima A, Stingaci E, Vlad L, Shvets N, Kandemirli F, Dimogloa A, Reynolds R.

Synthesis of novel 5-aryl-2-thio-1,3,4-oxadiazoles and the study of their structure-anti-mycobacterial activities.

Bioorg Med Chem. 2005; 13: 4842-4850.

doi:10.1016/j.bmc.2005.05.011

[14] Zarghi A, Tabatabai SA, Faizi M, Ahadian A, Navabi P, Zanganeh V, Shafiee A.

Synthesis and anticonvulsant activity of new 2-substituted-5-(2-benzyloxyphenyl)-1,3,4-oxadiazoles. Bioorg Med Chem Lett. 2005; 15: 1863-1865.

doi:10.1016/j.bmcl.2005.02.014

[15] Alafeefy AM.

Synthesis and antimicrobial activity of some new quinazolin-4(3H)-one derivatives.

Pharm Biol. 2008; 46: 751-756.

doi:10.1080/13880200802315907

[16] Sen Gupta AK, Misra HK.

Synthesis and evaluation of substituted quinazolone derivatives for antibacterial, antifungal, and antiacetylcholinesterase activities.

J Pharm Sci. 1980; 69: 1313-1317.

doi:10.1002/jps.2600691120

[17] Alagarsamy V, Murugesan S, Sheorey RV.

Synthesis and pharmacological investigation of novel 3-(benzyl)-2-substituted amino-3H-quinazolin-4ones as analgesic and anti-inflammatory agents.

Med Chem Res. 2008; 17: 564-577.

doi:10.1007/s00044-008-9098-z

[18] Jessy EM, Sambanthan AT, Alex J, Sridevi CH, Srinivasan KK.

Synthesis and biological evaluation of some novel quinazolones.

Indian J Pharm Sci. 2007; 69: 476-478.

[19] Shukla JS, Agarwal K, Rastogi R.

Synthesis of some new 2-substituted 3-[4-(N-arylsulphonylbiguanido)phenyl]quinazolin-4-one

hydrochlorides as potential anthelminthic agents.

Arch Pharm. 1983; 316: 525-529.

doi:10.1002/ardp.19833160610 
[20] Khalil AA, Abdel Hamide SG, Al-Obaid AM, El-Subbagh HI.

Substituted quinazolines, part 2. synthesis and in vitro anticancer evaluation of new 2-substituted mercapto-3H-quinazoline analogs.

Arch Pharm. 2003; 336: 95-103.

doi:0365-6233/02/0095

[21] Laddha SS, Bhatnagar SP.

Rapid microwave-assisted solution phase synthesis of 6,8-disubstituted 2-phenyl-3-

(substitutedbenzothiazol-2-yl)-4-[3H]-quinazolinones as novel anticonvulsants.

Phosphorus Sulfur and Silicon. 2008; 183: 2262-2273.

doi:10.1080/10426500801957766

[22] Alagarsamy V, Prabakaran L, Murugan RD, Gurumurth G, Bindu P, Arunkumar M, Bothiraja C. Synthesis and antihistaminic activity of some novel 2-mercapto-3-(substitutedmethylamino)quinazolin$4(3 H)$-ones.

Acta Pharm Turcica. 2000; 42: 33-38.

[23] Alagarsamy V, Murugesan S, Dhanabal K, Murugan M, Clercq E.

Anti-HIV, antibacterial and antifungal activities of some novel 2-methyl-3-(substituted methylamino)-

(3H)-quinazolin-4-ones.

Indian J Pharm Sci. 2007; 69: 304-307.

[24] Raffa D, Daidone G, Maggio B, Schillaci D, Plescia F.

Synthesis and antiproliferative activity of novel 3-(indazol-3-yl)-quinazolin-4(3H)-one and 3-(indazol-3yl)-benzotriazin-4(3H)-one derivatives.

Arch Pharm. 1999; 332: 317-320.

doi:10.1002/(SICl)1521-4184(19999)332:9<317::AID-ARDP317>3.0.CO;2-R

[25] Mosaad SM, Mohammed KI, Ahmed MA, Abdel-Hamide SG.

Synthesis of certain new 6 -iodoquinazolines as potential antitubercular agents.

J Applied Sci. 2004; 4: 302-307.

[26] Selvam P, Babu K, Padamraj R, Persoons L, Clercq E.

Synthesis, antiviral and cytotoxic activities of some novel 2-phenyl-3-disubstituted quinazolin-4(3H)ones.

African J Pharm Pharmacol. 2008; 2: 110-115.

[27] Joshi KC, Singh VK, Mehta DS, Sharma RC, Gupta L.

Fluorinated quinazolones iii: synthesis and CNS depressant activity of fluorinated quinazolone derivatives.

J Pharm Sci. 1975; 64: 1428-1430.

doi:10.1002/jps.2600640843

[28] Raffa D, Edler MC, Daidone G, Maggio B, Merickech M, Plescia S, Schillaci D, Bai R, Hamel E. Synthesis, cytotoxicity, and inhibitory effects on tubulin polymerization of a new 3-heterocyclo substituted 2-styrylquinazolinones.

Eur J Med Chem. 2004; 39: 299-304.

doi:10.1016/j.ejmech.2003.12.009

[29] Maarouf AR, El-Bendary ER, Goda FE.

Synthesis and evaluation of some novel quinazolinone derivatives as diuretic agents.

Arch Pharm. 2004; 337: 527-532.

doi:10.1002/ardp.200400869

[30] Kurogi Y, Inoue Y, Tsutsumi K, Nakamura S, Nagao K, Yoshitsugu H, Tsuda Y.

Synthesis and hypolipidemic activities of novel 2-[4-[(diethoxyphosphoryl)methyl]phenyl]quinazolines and $4(3 H)$-quinazolinones.

J Med Chem. 1996; 39: 1433-1437.

doi:10.1021/jm9506938

[31] Furniss BS, Hannaford AJ, Smith PWG, Tatchell AR.

In: Vogel's Textbook of Practical Organic Chemistry. 5th ed.

New York: Wiley, 1989, 692. 
[32] Katritzky AR, Vvedensky V, Cai X, Rogovoy B, Steel PJ.

Syntheses of 5-(2-arylazenyl)-1,2,4-triazoles and 2-amino-5-aryl-1,3,4-oxadiazoles. Arkivoc. 2002; 6: 82-90.

[33] Patel NB, Chaudhari RC.

Quinazolin-4(3H)-ones of 2-[(2',6'-dichlorophenyl)amino]phenyl acetic acid with substituted aryl acetamide and their microbial studies. J Ind Chem Soc. 2006; 83: 838-841.

[34] Patel NB, Lilakar JD.

Synthesis of new substituted $4(3 \mathrm{H})$-quinazolinones and their antibacterial activity. Ind J Heterocycl Chem. 2001; 11: 85-86.

[35] Rattan A.

In: Antimicrobials in laboratory medicine. New Delhi: Churchill BI, Livingstone, 2000, 85. 